The Kontsevich Weight of a Wheel with Spokes Pointing Outward Non Peer-reviewed author version

VAN DEN BERGH, Michel (2009) The Kontsevich Weight of a Wheel with Spokes

Pointing Outward. In: ALGEBRAS AND REPRESENTATION THEORY, 12(2-5). p. 443-479.

DOI: $10.1007 / \mathrm{s} 10468-009-9161-6$

Handle: http://hdl.handle.net/1942/9843 


\title{
THE KONTSEVICH WEIGHT OF A WHEEL WITH SPOKES POINTING OUTWARD
}

\author{
MICHEL VAN DEN BERGH
}

Dedicated to Fred Van Oystaeyen on the occasion of his 60th birthday.

\begin{abstract}
This is a companion note to "Hochschild cohomology and Atiyah classes" by Damien Calaque and the author. Using elementary methods we compute the Kontsevich weight of a wheel with spokes pointing outward. The result is in terms of modified Bernoulli numbers. The same result had been obtained earlier by Torossian (unpublished) and also recently by Thomas Willwacher using more advanced methods.
\end{abstract}

\section{Contents}

1. Introduction and statement of the main result 1

2. Acknowledgment 3

3. Graphical representation of the computation 3

4. A recursion relation for $\alpha_{n, m} \quad 14$

5. A recursion relation for $\beta_{n, m} \quad 16$

6. A recursion relation for $\bar{\beta}_{n, m} \quad 21$

7. A recursion not involving unknown quantities. 23

8. A formula for $w_{n} \quad 24$

9. Solving the recursion 26

References 30

\section{IntroduCtion AND STATEMENT OF THE MAIN RESUlT}

This is a companion note to [2]. We refer to $[2, \S 8]$ and [7] for unexplained notations and conventions.

1991 Mathematics Subject Classification. Primary 14F99, 14D99.

Key words and phrases. Kontsevich weight.

The author is a director of research at the FWO. 
Below we will compute the Kontsevich weight $w_{n}$ of the "opposite" wheel

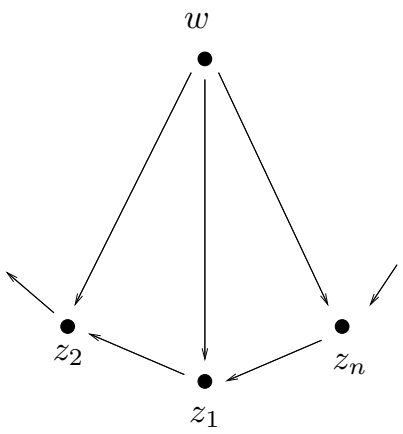

with edge ordering $\left(z_{1}, z_{2}\right)<\cdots<\left(z_{n}, z_{1}\right)<\left(w, z_{1}\right)<\cdots<\left(w, z_{n}\right)$.

Thus ${ }^{1}$

$$
w_{n}=\frac{1}{(2 \pi)^{2 n}} \int_{C_{n+1,0}} d \varphi\left(z_{1}, z_{2}\right) \cdots d \varphi\left(z_{1}, z_{n}\right) d \varphi\left(w, z_{1}\right) \cdots d \varphi\left(w, z_{n}\right)
$$

where $\varphi(u, v)$ is computed as in the following diagram.

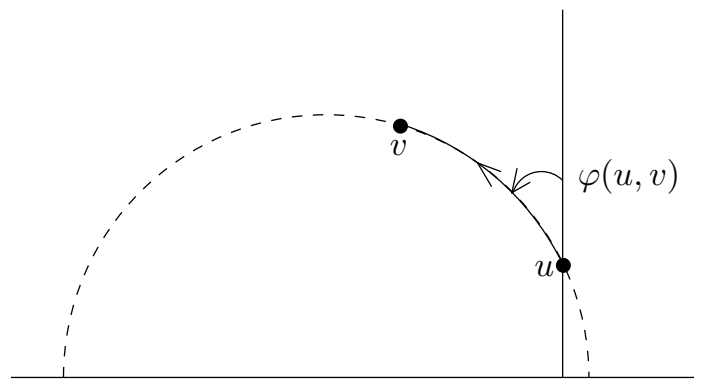

and where $C_{n+1,0}$ is the configuration space of $n+1$ points in the complex upper half plane $\mathcal{H}$. If we normalize things by putting $w=i$ then the integration domain is equal to the complement of the big diagonal in $(\mathcal{H}-\{i\})^{n}$.

Recall (e.g. [12]) that the modified Bernoulli numbers $\beta_{n}$ are defined by

$$
\begin{aligned}
\sum_{n} \beta_{n} x^{n} & =\frac{1}{2} \log \frac{e^{x / 2}-e^{-x / 2}}{x} \\
& =\frac{x^{2}}{48}-\frac{x^{4}}{5760}+\frac{x^{6}}{362880}-\frac{x^{8}}{19353600}+\frac{x^{10}}{958003200}-\frac{691 x^{12}}{31384184832000}+\cdots
\end{aligned}
$$

The main result of this note is a relatively elementary proof of the following (see $\S 9.6)$

Proposition 1.1. [13, Theorem 18]

$$
w_{n}=-(-1)^{n(n-1) / 2} n \beta_{n}
$$

We needed (1.1) for the proof of [2, Thm 1.3]. At that time we didn't have a reference so we did the computation ourselves. After this note was posted on the arXiv Torossian sent us an argument which shows that (1.1) follows from the work of Cattaneo and Felder [4,5] on the quantization of coisotropic manifolds combined with Shoikhet's vanishing results for wheels with spokes pointing inwards

\footnotetext{
${ }^{1}$ This is the traditional Kontsevich weight. In $[2, \S 8]$ we use $W_{n}=(-1)^{2 n(2 n-1) / 2} w_{n}=$ $(-1)^{n} w_{n}$. Since $w_{n}=0$ for $n$ odd we actually have $W_{n}=w_{n}$.
} 
[11] and some facts related to the Campbell-Baker-Hausdorff formula. In this way one obtains a shorter and more conceptual proof of (1.1). Torossian also pointed out Willwacher's recent work [13] which uses the same ingredients.

While more conceptual it seems this approach is substantially less elementary than ours so we think that this note is still useful.

Let us finally also mention that a result similar to (1.1) was announced by Shoikhet in $[11, \S 2.3 .1]$. It can presumably be obtained from the methods in [10].

The structure of this note is as follows: in $\S 3$ we represent our computations graphically. This makes it easy to see which boundary components in Stokes theorem yield non-trivial contributions. We ignore signs as these are not easy to handle graphically.

In $\S 4-\S 8$ we compute the contributions of the relevant boundary components with precise signs. Thanks to some "lucky" cancellations we obtain a system of recursions for computing $w_{n}$.

Finally in $\S 9$ we solve the recursions.

The reader will notice that some formulas and figures appear more than once in this paper. This has been done to make the three logical parts $\S 3, \S 4-\S 8, \S 9$ more or less independently readable. This should make our computations easier to follow.

As indicated above we use almost nothing but Stokes theorem in our computations. For a general introduction to the use of Stokes theorem in the computation of Kontsevich weights see Torossian's article in [3]

\section{Acknowledgment}

The symbolic computations were done using the SAGE [9] interface to the computer algebra package Maxima [8]. Many identities and integrals were tested numerically using the Monte Carlo integration library which is part of GSL [6].

We thank Charles Torossian for pointing how (1.1) follows from the work of Cattaneo and Felder.

Finally I would like to specially thank the anonymous referee for his very careful reading of the manuscript. This has allowed me to improve the exposition and to correct numerous "misprints".

\section{Graphical Representation of the computation}

In this section we give a graphical overview of our computations. We will ignore signs. Hence we don't care about orientations or the ordering of the factors in a product of forms.

3.1. Graphical language. In the Kontsevich integral we associate to a graph $\Gamma$ a form $\omega_{\Gamma}$ on a suitable configuration space $C_{p, q}^{+}$. The form $\omega_{\Gamma}$ is the product of $d \varphi(u, v)$, where $(u, v)$ runs over the edges of $\Gamma$, divided by $(2 \pi)^{e}$ where $e$ is the number of edges.

Besides the usual Kontsevich graphs we introduce graphs with some new kind of arrows.

An arrow of the form

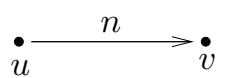

corresponds to a factor $d\left(\varphi(u, v)^{n}\right) /(2 \pi)^{n}$ in $\omega_{\Gamma}$. For such an arrow we assume that we have made a branch cut such that $\varphi(u, v) \in] 0,2 \pi[$. I.e. if $\operatorname{Re} u=\operatorname{Re} v$ 
then $\operatorname{Im} v<\operatorname{Im} u$. Note that we have to specify the branch cut as the value of the integral depends on it.

A bold arrow of the form

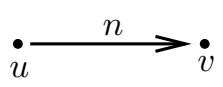

corresponds to a factor $\varphi(u, v)^{n} /(2 \pi)^{n}$ in $\omega_{\Gamma}$. We make the same branch cut as above. If $n$ is not indicated then we assume $n=1$.

We introduce a third kind of special arrow. A dashed arrow of the form

$$
\dot{u}--\rightarrow \dot{v}
$$

indicates a restriction of the integration domain to $\operatorname{Re} v=\operatorname{Re} u+\epsilon, \operatorname{Im} v>\operatorname{Im} u$ with $\epsilon$ a positive infinitesimal.

Here is the basic computation rule for computing $\int \omega_{\Gamma}$ for one of the generalized graphs (Rule 1).

$$
\begin{aligned}
& \int \dot{u} \stackrel{n}{u} \dot{v}= \pm \int_{\partial} \dot{u} \stackrel{n}{\longrightarrow} \dot{v}
\end{aligned}
$$

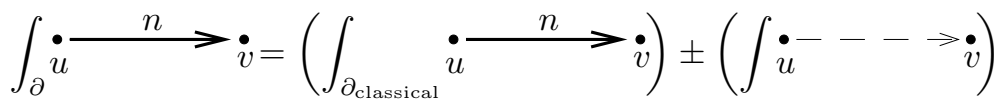

Here $\partial$ denotes the boundary of the integration domain (including the branch cut). $\partial_{\text {classical }}$ denotes the boundary without the branch cut. $\partial_{\text {classical }}$ can be determined combinatorially by contracting vertices in $\Gamma$ and moving vertices to the real line.

The difference between $\partial$ and $\partial_{\text {classical }}$ is given by the two sides of the branch cut. The difference in value of $\varphi(u, v)$ on opposite sides of the branch cut is $2 \pi$. This gives the contribution with the dashed arrow.

Here is another obvious computation rule (Rule 2)

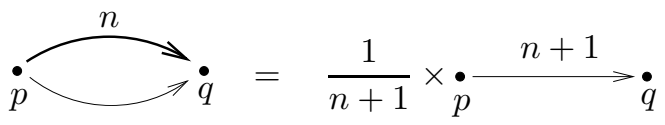

Below we will also consider some integrals over the configuration spaces $C_{p}$ consisting of $p$ points in the complex plane. These are it terms of $\theta(u, v)$ where $\theta(u, v)$ is as in the following diagram

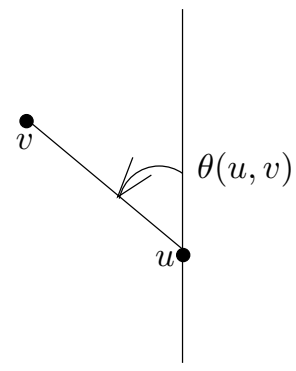

To indicate that we are integrating over some $C_{p}$ we will put the graph in a box. Otherwise we use the same conventions as above (with $\theta(u, v)$ replacing $\varphi(u, v)$ ). Since $d \theta(u, v)=d \theta(v, u)$ some arrow indicators are superfluous. We will omit those.

We will repeatedly use the following vanishing result

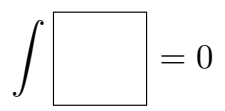


provided the graph in the box contains only ordinary edges and the number of edges is $\geq 3$. An integral as in (3.1) often arises as a factor of an integral over a boundary component of $C_{n+1,0}$.

3.2. Applying Stokes theorem to $w_{n}$. We will now apply this graphical language to computations with wheels. We assume first $n \geq 2$
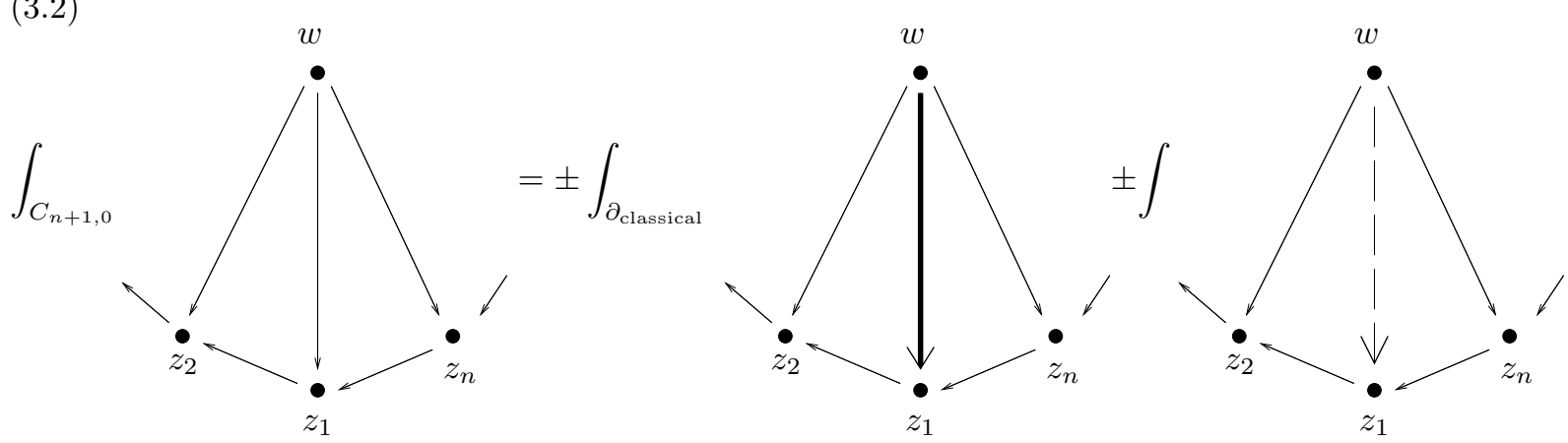

We now simplify the integral over $\partial_{\text {classical }}$ by looking at the different components of $\partial_{\text {classical }}$.

(1) A group of vertices moves to the real line. Since every vertex has a least one normal outgoing arrow this means that to obtain a non-zero result all vertices should move to the real line which is excluded. So components of this type contribute nothing.

(2) A group of vertices $S$ comes together. Assume first $w \in S$. If $z_{i} \in S, i<n$ then to avoid double arrows after contraction we should also have $z_{i+1} \in S$. If $z_{1} \in S$ this means we have to contract all vertices. I.e. the integral over this component becomes an integral over $C_{n+1}$

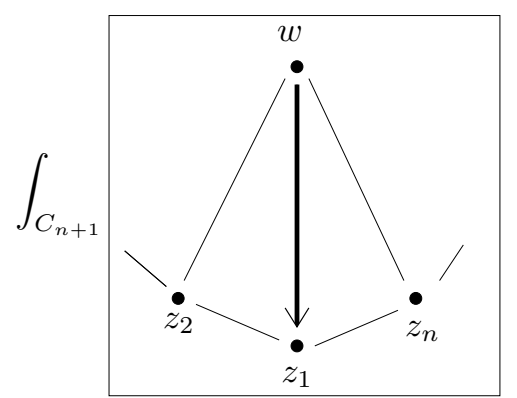

(3) Assume $z_{1} \notin S$. If $z_{i} \in S$ for $i<n$ then we contract more than one edge and the resulting integral is zero by (3.1). Thus $S=\left\{w, z_{n}\right\}$. The resulting 
integral is (using Rule 2)

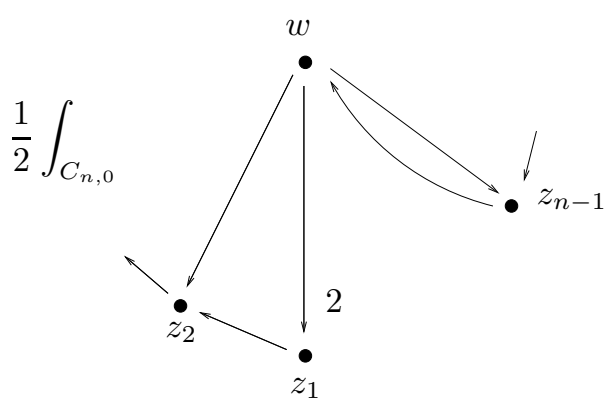

(4) Now assume $w \notin S$. This means that we contract an edge $\left(z_{i}, z_{i+1}\right)$ or $\left(z_{n}, z_{1}\right)$ (if we contract more edges then the integral is zero by (3.1)). The only contractions which do not create double edges are $\left(z_{1}, z_{2}\right)$ and $\left(z_{n}, z_{1}\right)$. In that case the resulting integrals are (using Rule 2)

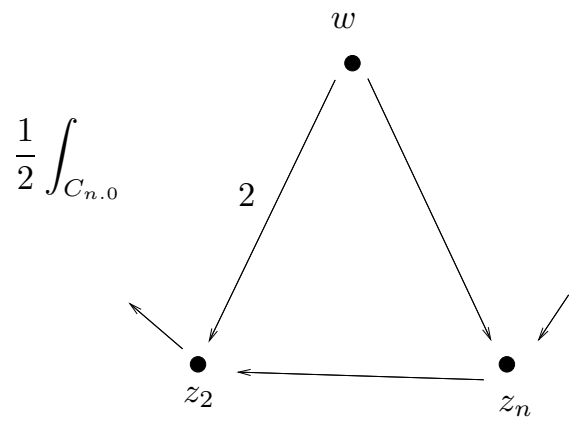

and

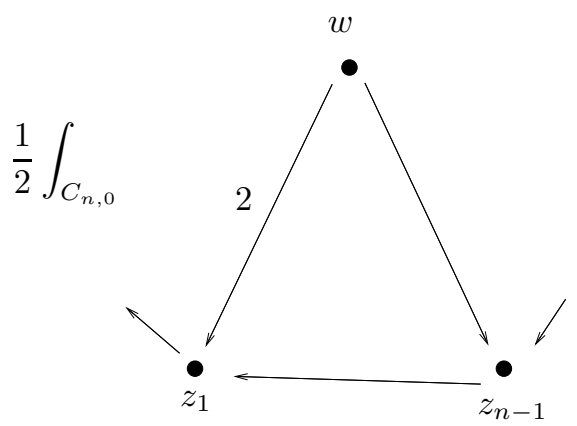

3.3. The boundary component associated to the branch cut. We need to compute the integral associated to the graph with the dashed arrow in (3.2). In order to derive a recursion relation we work more generally, starting with the following 
identity.

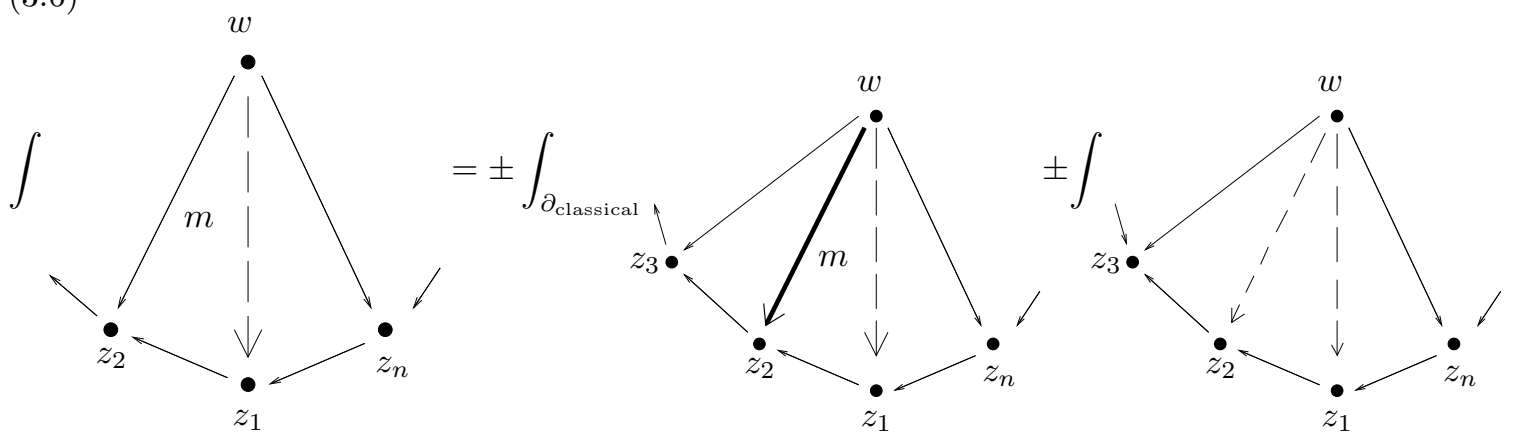

However the integral containing the two dashed arrows is zero since if $z_{1}, z_{2}$ move on the same vertical line then $d \varphi\left(z_{1}, z_{2}\right)=0$.

It remains to determine the integral over $\partial_{\text {classical }}$. We consider the various boundary components. We now assume $n \geq 3$.

(1) A group of vertices moves to the real line. Since every vertex has at least one normal outgoing arrow (using $n \geq 2$ ) this means that to obtain a nonzero result all vertices should move to the real line which is excluded. So no contribution from these components.

(2) A group of vertices $S$ comes together. Assume first $w \in S$. If $z_{i} \in S$, $i \neq 1, n$ then to avoid double arrows after contraction we should also have $z_{i+1} \in S$. However if $z_{2} \in S$ or $z_{n} \in S$ then $z_{1} \in S$ for otherwise after contraction a solid and a dashed arrow coincide and thus the result is zero. Thus we either contract all vertices or we contract $w, z_{i}, \ldots, z_{n}, z_{1}$ for $i \geq 3$ or we contract $\left(w, z_{1}\right)$. If we contract all vertices then the integral becomes

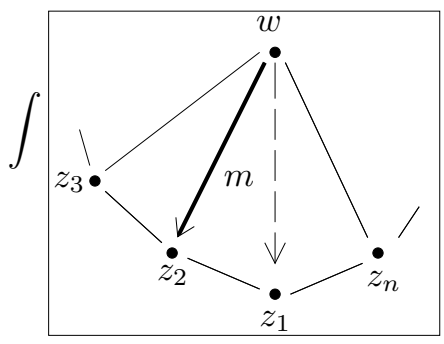

(3) Assume we contract $w, z_{i}, \ldots, z_{n}, z_{1}$ for $3 \leq i \leq n$. Then the resulting integral is a multiple of

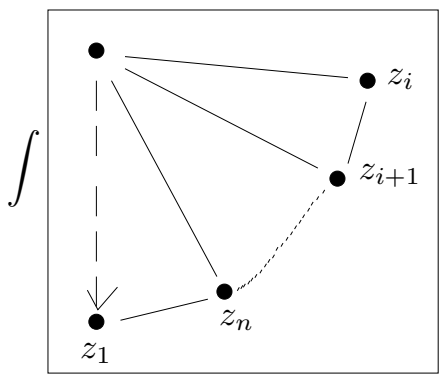


(4) It remains to consider the case $S=\left\{z_{1}, w\right\}$. In that case we get using Rule 2 :

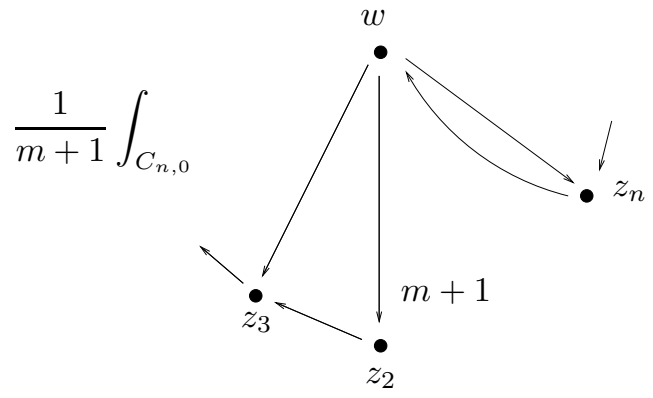

(5) Now assume $w \notin S$. This means that we contract an edge $\left(z_{i}, z_{i+1}\right)$ or $\left(z_{n}, z_{1}\right)$ (if we contract more edges then the integral is zero by $(3.1)$ ). The only two possibilities which do not give zero immediately are $\left(z_{1}, z_{2}\right)$ and $\left(z_{2}, z_{3}\right)$. The resulting integrals are

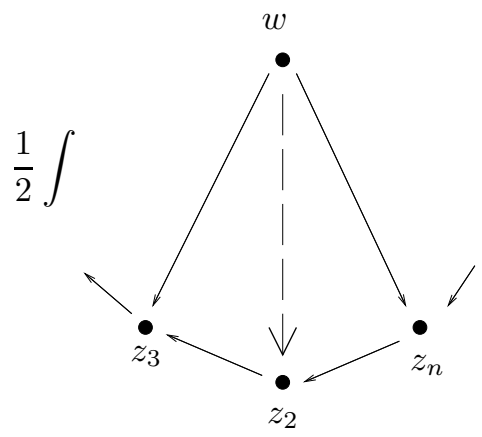

and (using Rule 2)

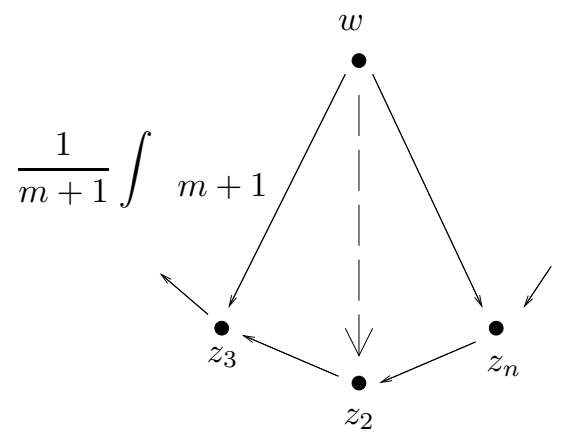

The factor $\frac{1}{2}$ in the first integral comes from the fact that in this boundary component we have $\varphi\left(w, z_{2}\right)=0$ if $z_{2}$ is to the left of $z_{1}$. 
As will be explained below we need a second method for attacking the term with the dashed arrow in (3.2). Therefore we use

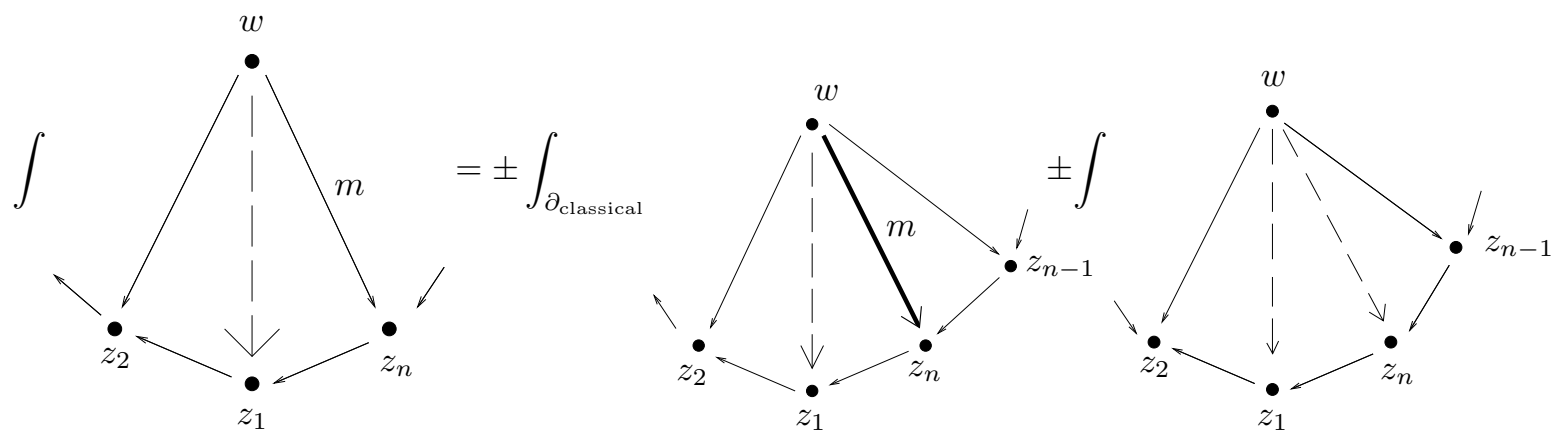

Again the integral containing the two dashed arrows is zero. Likewise is the integral over the boundary components where some vertices move to the real line. We discuss the other boundary components of $\partial_{\text {classical }}$ assuming $n \geq 3$.

(1) A group of vertices $S$ comes together. Assume first $w \in S$. If $z_{i} \in S$, $1 \leq i<n-1$ then to avoid double arrows after contraction we should also have $z_{i+1} \in S$. However if $z_{n} \in S$ of $z_{2} \in S$ then $z_{1} \in S$ for otherwise after contraction a solid and a dashed arrow coincide and thus the result is zero.

It follows that the possibilities for contraction are $\left\{z_{1}, \ldots, z_{n}, w\right\},\left\{z_{1}, \ldots, z_{n-1}, w\right\}$ or $\left\{z_{i}, \ldots, z_{n-1}, w\right\}$ for $i \geq 3$. In the last case we must have $i=n-1$ by (3.1).

If we contract all vertices then the contribution to the integral is

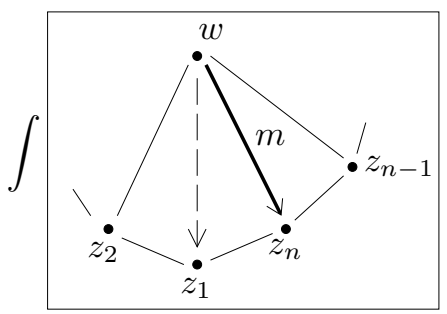

(2) If we contract $w, z_{1}, \ldots, z_{n-1}$ then the resulting integral is a multiple of

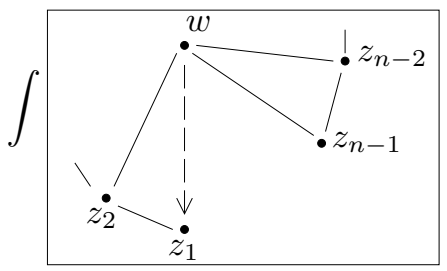


(3) It remains to consider the case $S=\left\{z_{n-1}, w\right\}$. In that case we obtain (using Rule 2)

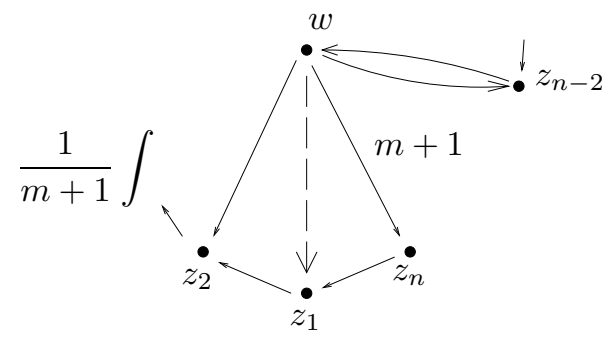

(4) Now assume $w \notin S$. This means that we contract an edge $\left(z_{n}, z_{1}\right)$ or $\left(z_{n-1}, z_{n}\right)$ (otherwise the result is zero by (3.1) or by the fact that we create double arrows). The resulting integrals are

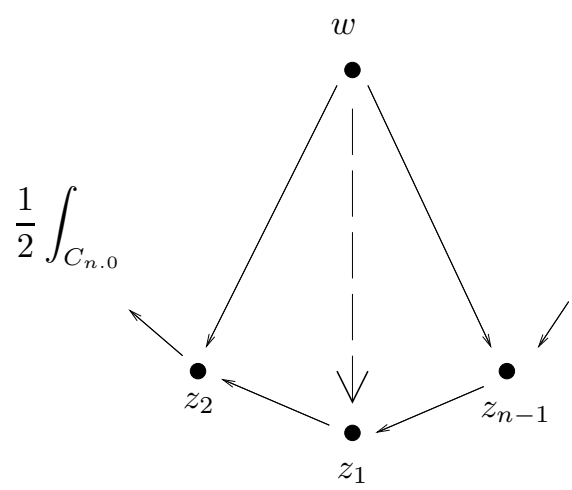

and (using Rule 2)

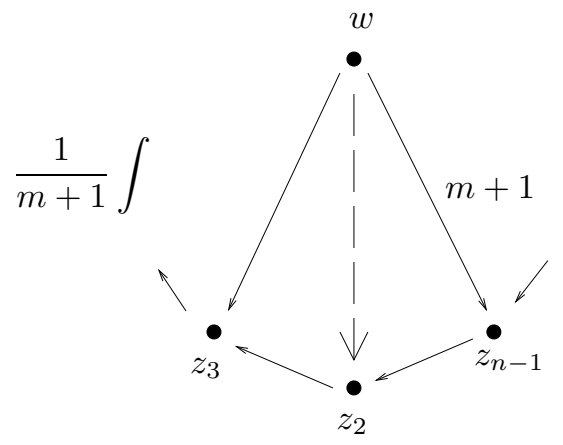

The factor $1 / 2$ in the first integral comes from the fact that due to our branch cut $\varphi\left(w, z_{n}\right)=0$ if $z_{2}$ is to the left of $z_{1}$.

3.4. Further comments. We now indicate how the relations we have derived will be used. We still do not specify precise signs. 
(1) We first discuss the partial evaluation of $w_{n}$ using (3.2). One ingredient that enters is (3.3). We define

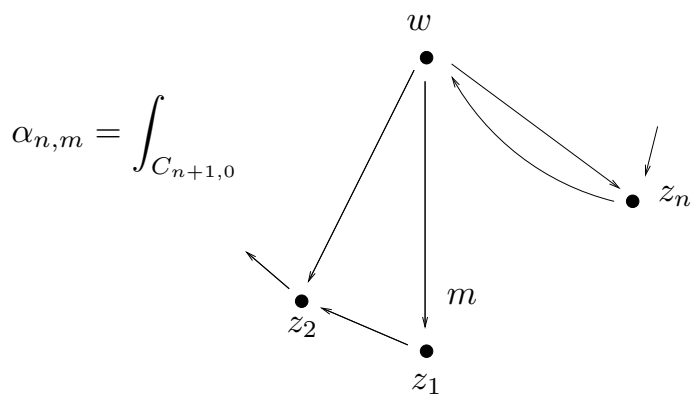

We will evaluate $\alpha_{n, m}$ by subsequently integrating over $z_{1}$, $z_{2}$, etc... See $\S 4$ below.

(2) Another ingredient that enters is (8.2).

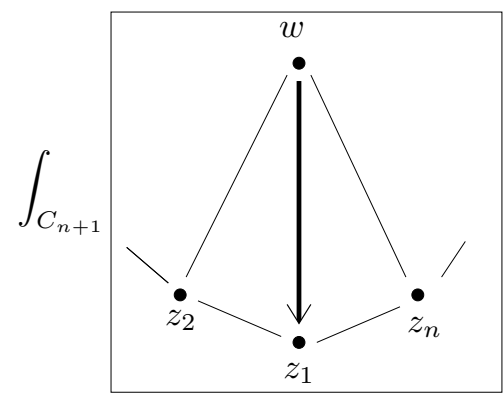

We do not know how to evaluate this integral in general but we will show by a symmetry argument that it is zero if $n$ is even (see $\S 8.2$ ).

(3) The final ingredient is given by the two identically looking integrals

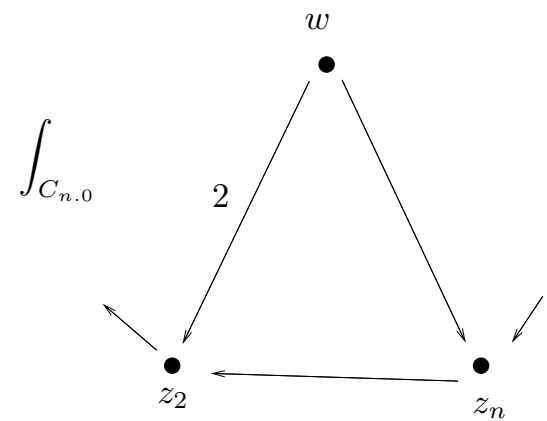

and

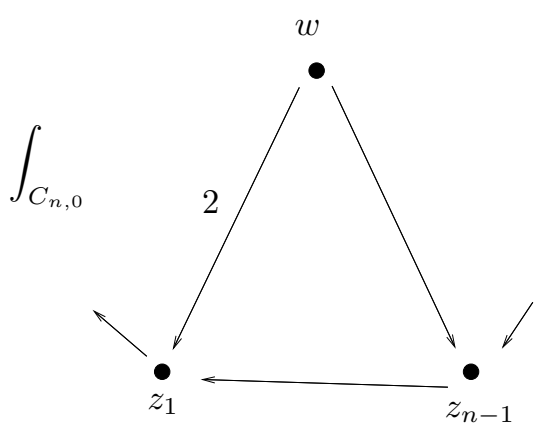


We will show that the signs are such that these two integrals cancel (see $\S 8.3)$.

(4) So ultimately we get an expression for $w_{n}$ in terms of $\alpha_{n-1, m+1}$ (which we know how to compute) and

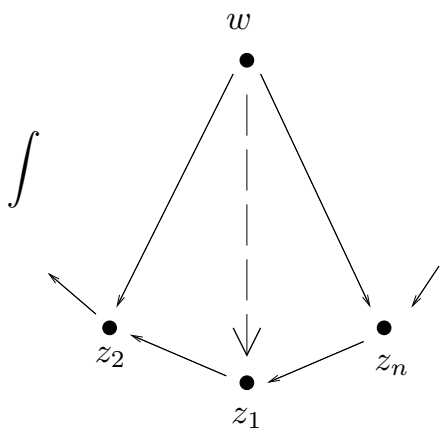

which is a special case of both the following integrals

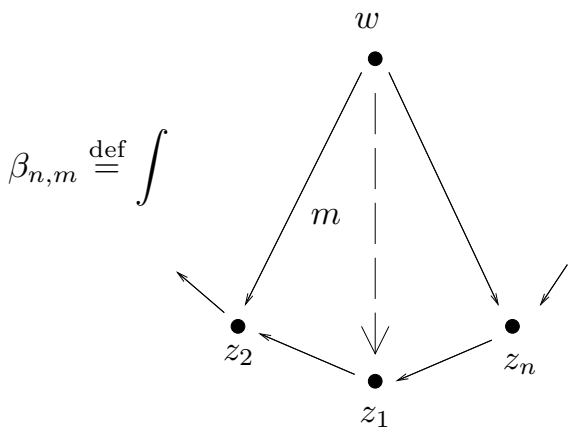

and

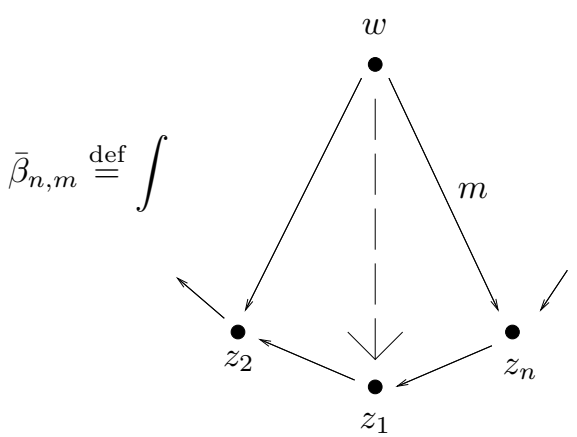

(5) We now discuss $\beta_{n, m}$ using (3.6). We will show below (see $\S 5.2$ ) that

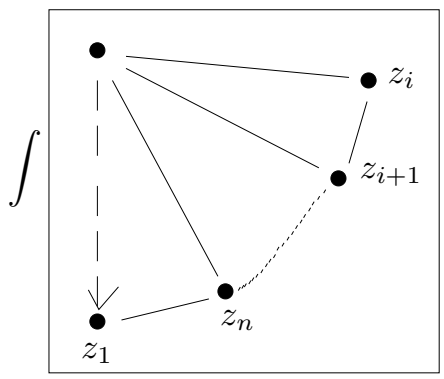


is zero. Hence we obtain an expression for $\beta_{n, m}$ in terms of $\alpha_{n-1, m+1}$, $\beta_{n-1,1}, \beta_{n+1, m-1}$ and

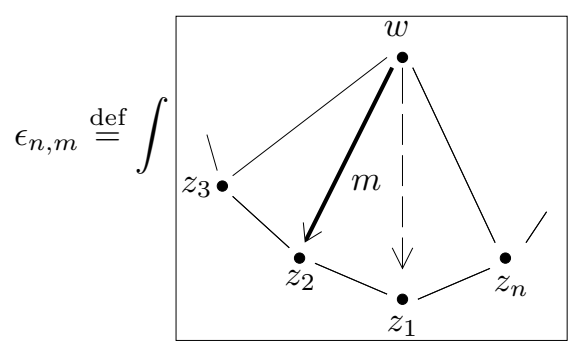

(6) In a similar way we discuss $\bar{\beta}_{n, m}$. As already pointed out we will show that the integral

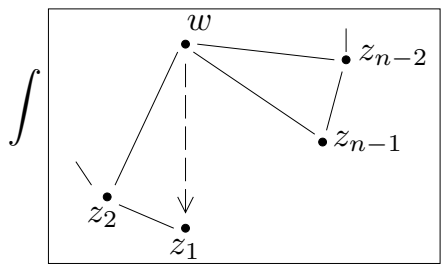

is zero (see $§ 5.2$ below). We will also show that the integral

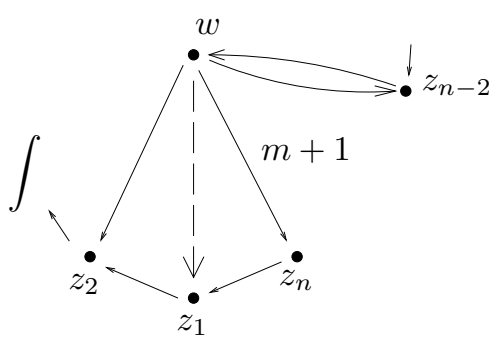

is zero (see $\S 6.1)$.

As a result we obtain an expression for $\bar{\beta}_{n, m}$ in terms of $\bar{\beta}_{n-1, m+1}, \beta_{n-1,1}$ and

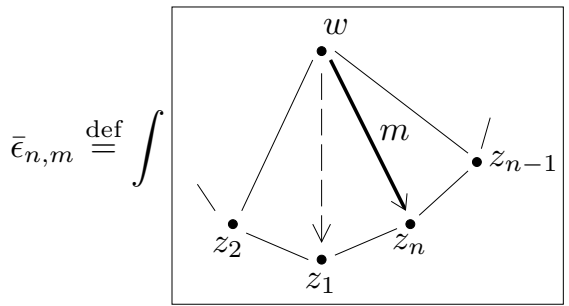

(7) We will show below by a symmetry argument that $\epsilon_{n, m}=-\bar{\epsilon}_{n, m}$ (see $\S 7.1$ ).

(8) Unfortunately we do not know how to compute $\epsilon_{n, m}$. However we will show below that by considering a suitable linear combination of $\beta_{n, m}$ and $\bar{\beta}_{n, m}$ the unknown quantity $\epsilon_{n, m}$ cancels out in the computation. This will ultimately give us a recursive procedure for computing $w_{n}$. 


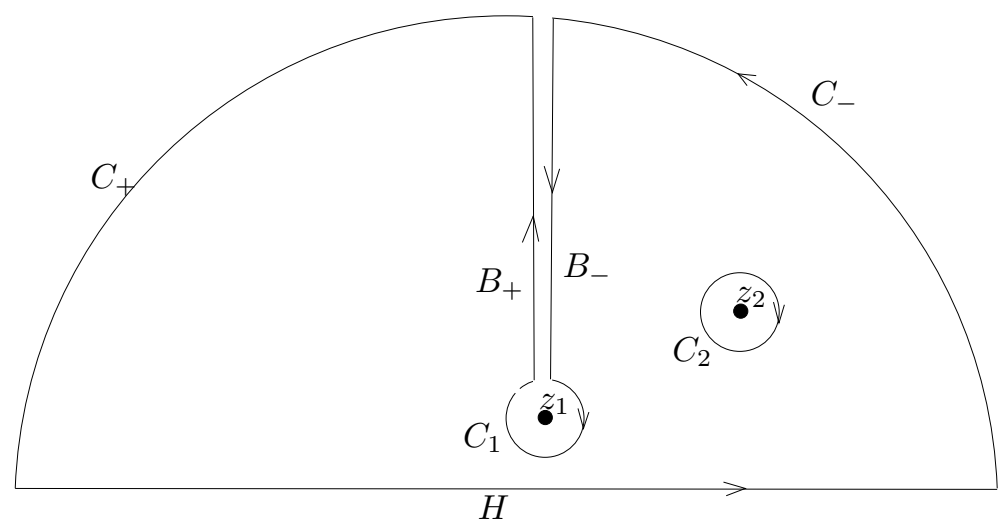

FigURE 1

\section{A RECURSion RELATiOn FOR $\alpha_{n, m}$}

By our definition in $\S 3.4, \alpha_{n, m}$ is the integral associated to the enhanced graph

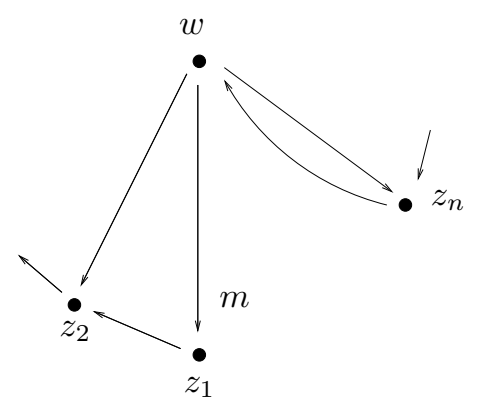

Thus with our standard ordering of edges we have

$\alpha_{n, m}=\frac{1}{(2 \pi)^{2 n+m-1}} \int_{C_{n+1,0}} d \varphi\left(z_{1}, z_{2}\right) \cdots d \varphi\left(z_{n-1}, z_{n}\right) d \varphi\left(z_{n}, w\right) d \varphi\left(w, z_{1}\right)^{m} d \varphi\left(w, z_{2}\right) \cdots d \varphi\left(w, z_{n}\right)$

In this section we prove

$$
\alpha_{1, m}=-\left(\frac{1}{2}-\frac{1}{m+1}\right)
$$

and for $n \geq 2$.

$$
\alpha_{n, m}=(-1)^{n}\left(\frac{1}{2} \alpha_{n-1,2}-\frac{1}{m+1} \alpha_{n-1, m+1}\right)
$$

We put $\alpha_{0, m}=1$ such that (4.2) holds for $n \geq 1$.

4.1. Step 1. We will prove

$$
\int_{z \in \mathcal{H} \backslash\left\{z_{1}, z_{2}\right\}} d \varphi\left(z_{1}, z\right)^{m} d \varphi\left(z, z_{2}\right)=(2 \pi)^{m} \varphi\left(z_{1}, z_{2}\right)-2 \pi \varphi\left(z_{1}, z_{2}\right)^{m}
$$

where we have made a branch cut such that $\left.\varphi\left(z_{1}, z\right) \in\right] 0,2 \pi[$.

In Figure 1 we have indicate the integration domain and its boundary. Here 


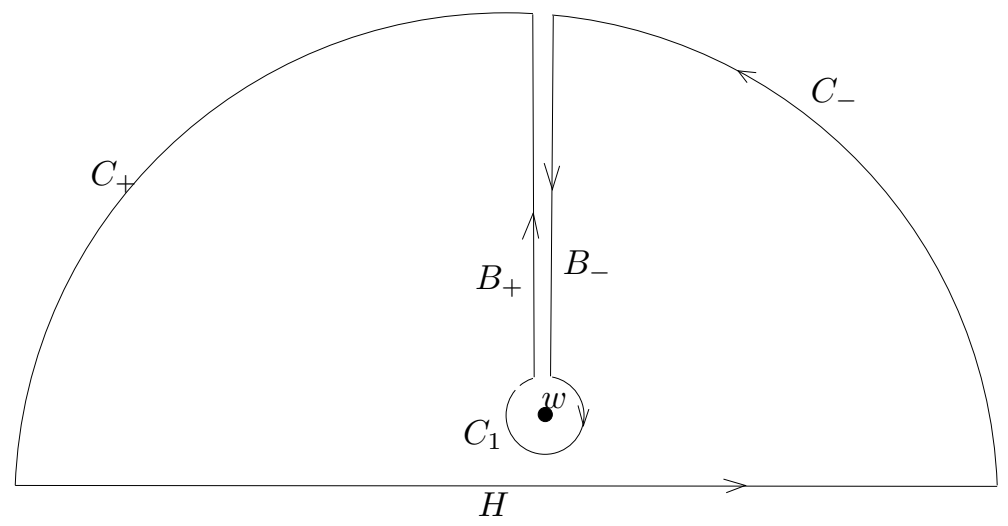

FiguRE 2

$C_{+} \cup C_{-}$is the circle at infinity, $B_{+}, B_{-}$are infinitesimally close together and $C_{1}$, $C_{2}$ have infinitesimal radius. Finally $H$ is the real line. Thus we have

$$
\int_{z \in \mathbb{C} \backslash\left\{z_{1}, z_{2}\right\}} d \varphi\left(z_{1}, z\right)^{m} d \varphi\left(z, z_{2}\right)=\int_{\text {boundary }} \varphi\left(z_{1}, z\right)^{m} d \varphi\left(z, z_{2}\right)
$$

We first compute the integral over $C_{ \pm} \cup B_{ \pm} \cup C_{1} \cup H$. On $B_{-} \cup C_{-}$we have that $\varphi\left(z_{1}, z\right)$ is constant and equal to $2 \pi$. On $B_{+} \cup C_{+}$we have that $\varphi\left(z_{1}, z\right)=0$. On $H \cup C_{1}$ we have that $d \varphi\left(z, z_{2}\right)=0$.

Thus we find

$$
\int_{B_{ \pm} \cup C_{ \pm} \cup C_{1} \cup H} \varphi\left(z_{1}, z\right)^{m} d \varphi\left(z, z_{2}\right)=(2 \pi)^{m} \int_{B_{-} \cup C_{-}} d \varphi\left(z, z_{2}\right)=(2 \pi)^{m} \varphi\left(z_{1}, z_{2}\right)
$$

The integral over $C_{2}$ is equal to $-2 \pi \varphi\left(z_{1}, z_{2}\right)^{m}$. This finishes the proof of (4.3).

4.2. Step 2. We will prove

$$
\int_{z \in \mathcal{H} \backslash\{w\}} d \varphi(w, z)^{m} d \varphi(z, w)=(2 \pi)^{m+1}\left(\frac{1}{2}-\frac{1}{m+1}\right)
$$

In Figure 2 we have indicated the integration domain and its boundary. Thus we have

$$
\int_{z \in \mathbb{C} \backslash\{w\}} d \varphi(w, z)^{m} d \varphi(z, w)=\int_{\text {boundary }} \varphi(w, z)^{m} d \varphi(z, w)
$$

On $B_{-} \cup C_{-}$we have that $\varphi(w, z)$ is constant and equal to $2 \pi$. On $B_{+} \cup C_{+}$we have that $\varphi(w, z)=0$. On $H$ we have that $d \varphi(z, w)=0$. Thus

$$
\int_{C_{ \pm} \cup B_{ \pm} \cup H} \varphi(w, z)^{m} d \varphi(z, w)=(2 \pi)^{m} \pi
$$

It remains to compute

$$
\int_{C_{1}} \varphi(w, z)^{m} d \varphi(z, w)
$$

We put $z=w+r e^{-i \theta}$ for $\left.\theta \in\right]-\pi / 2,3 \pi / 2[$. We have $\varphi(w, z)=3 \pi / 2-\theta$ and hence $d \varphi(z, w)=-d \theta$. Thus we get

$$
\int_{C_{1}} \varphi(w, z)^{m} d \varphi(z, w)=-\int_{-\pi / 2}^{3 \pi / 2}(3 \pi / 2-\theta)^{m} d \theta=-\int_{0}^{2 \pi} \alpha^{m} d \alpha=-\frac{(2 \pi)^{m+1}}{m+1}
$$


MICHEL VAN DEN BERGH

This finishes the proof of (4.4).

4.3. Step 3. Now we prove (4.1).

$\alpha_{1, m}=\frac{1}{(2 \pi)^{m+1}} \int d \varphi\left(z_{1}, w\right) d \varphi\left(w, z_{1}\right)^{m}=-\frac{1}{(2 \pi)^{m+1}} \int d \varphi\left(w, z_{1}\right)^{m} d \varphi\left(z_{1}, w\right)=-\left(\frac{1}{2}-\frac{1}{m+1}\right)$ by (4.4).

4.4. Step 4. Now we prove (4.2).

$\alpha_{n, m}=(-1)^{n} \frac{1}{(2 \pi)^{2 n+m-1}} \int d \varphi\left(w, z_{1}\right)^{m} d \varphi\left(z_{1}, z_{2}\right) \cdots d \varphi\left(z_{n-1}, z_{n}\right) d \varphi\left(z_{n}, w\right) d \varphi\left(w, z_{2}\right) \cdots d \varphi\left(w, z_{n}\right)$

Integrating over $z_{1}$ and using (4.3) first we find

$$
\begin{aligned}
\alpha_{n, m}=(-1)^{n} \frac{1}{(2 \pi)^{2 n+m-1}} \int\left((2 \pi)^{m} \varphi\left(w, z_{2}\right)-2 \pi \varphi\left(w, z_{2}\right)^{m}\right) \times \\
\quad d \varphi\left(z_{2}, z_{3}\right) \cdots d \varphi\left(z_{n-1}, z_{n}\right) d \varphi\left(z_{n}, w\right) d \varphi\left(w, z_{2}\right) \cdots d \varphi\left(w, z_{n}\right)
\end{aligned}
$$

which is equal to

$$
\begin{aligned}
& \quad(-1)^{n} \frac{1}{2(2 \pi)^{2 n-1}} \int d \varphi\left(z_{2}, z_{3}\right) \cdots d \varphi\left(z_{n-1}, z_{n}\right) d \varphi\left(z_{n}, w\right) d \varphi\left(w, z_{2}\right)^{2} \cdots d \varphi\left(w, z_{n-1}\right) \\
& -(-1)^{n} \frac{1}{(m+1)(2 \pi)^{2 n+m-2}} \int d \varphi\left(z_{2}, z_{3}\right) \cdots d \varphi\left(z_{n-1}, z_{n}\right) d \varphi\left(z_{n}, w\right) d \varphi\left(w, z_{2}\right)^{m+1} \cdots d \varphi\left(w, z_{n}\right)
\end{aligned}
$$

So that we indeed find

$$
\alpha_{n, m}=(-1)^{n}\left(\frac{1}{2} \alpha_{n-1,2}-\frac{1}{m+1} \alpha_{n-1, m+1}\right)
$$

5. A Recursion Relation for $\beta_{n, m}$

By our definition in $\S 3.4, \beta_{n, m}$ is the integral associated to the enhanced graph

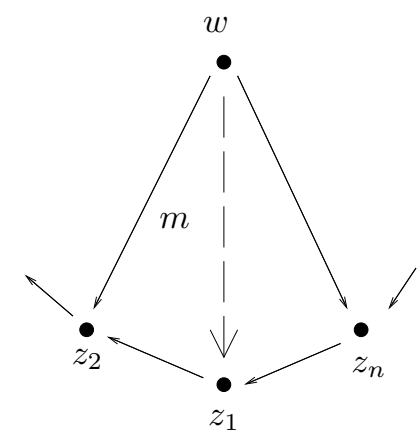

Thus with our standard edge ordering

$\beta_{n, m}=\frac{1}{(2 \pi)^{2 n+m-2}} \int_{B_{-} \backslash D} d \varphi\left(z_{1}, z_{2}\right) \cdots d \varphi\left(z_{n-1}, z_{n}\right) d \varphi\left(z_{n}, z_{1}\right) d \varphi\left(w, z_{2}\right)^{m} \cdots d \varphi\left(w, z_{n}\right)$

where

$$
\begin{aligned}
B_{-} & =\left\{\operatorname{Re} z_{1}=\operatorname{Re} w+, \operatorname{Im} z_{1}>\operatorname{Im} w\right\} \subset C_{n+1,0} \\
D & =\left\{\operatorname{Re} z_{2}=\operatorname{Re} w, \operatorname{Im} z_{2}>\operatorname{Im} w\right\} \subset C_{n+1,0}
\end{aligned}
$$

( $D$ represents the branch cut associated to the arrow $w \stackrel{m}{\longrightarrow} z_{2}$, see $\S 3.1$ ). We need to be careful about specifying the orientation of the integration domain. We do 
this next. We first normalize things by putting $w=i$. If $z_{j}=x_{j}+i y_{j}$ then $C_{n+1,0}$ is oriented by [1]

$$
d x_{1} d y_{1} d x_{2} d y_{2} \cdots d x_{n} d y_{n}
$$

In the neighborhood of $B_{-}$we have coordinates $\left(x_{1}, y_{1}, \ldots, x_{n}, y_{n}\right)$ with $x_{1} \geq 0$. Therefore if $B_{-}$is oriented with its outgoing normal (as is necessary for the application of Stokes theorem) then it is oriented by

$$
-d y_{1} d x_{2} d y_{2} \cdots d x_{n} d y_{n}
$$

Having determined the orientation of $B_{-}$we let $w$ be free again. In this section we will prove

$$
\beta_{2, m}=-\frac{1}{8}+\frac{1}{m+1}\left(\frac{1}{2}-\frac{1}{m+2}\right)
$$

and for $n \geq 3$

$\beta_{n, m}=-\frac{1}{m+1} \alpha_{n-1, m+1}+(-1)^{n+1} \frac{1}{2} \beta_{n-1,1}+(-1)^{n} \frac{1}{m+1} \beta_{n-1, m+1}+(-1)^{n} \epsilon_{n, m}$

where $\epsilon_{n, m}$ is the integral associated to the graph

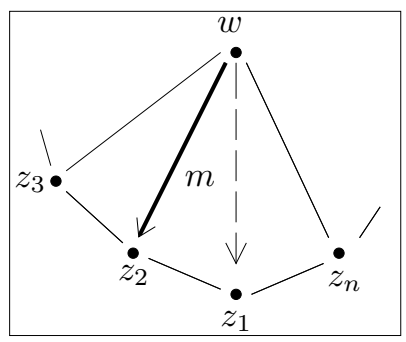

(see $§ 3.4)$. Thus with our standard orientation on edges

$$
\epsilon_{n, m}=\frac{1}{(2 \pi)^{2 n+m-2}} \int \theta\left(w, z_{2}\right)^{m} d \theta\left(z_{1}, z_{2}\right) d \theta\left(z_{2}, z_{3}\right) \cdots d \theta\left(z_{n}, z_{1}\right) d \theta\left(w, z_{3}\right) \cdots d \theta\left(w, z_{n}\right)
$$

Here we put $w=0, z_{1}=i$ and the integral is over the complement of the diagonal in $(\mathbb{C}-\{0, i\})^{n-1}$.

5.1. Step 1. We first consider the case $n=2$. Thus

$$
\beta_{2, m}=\frac{1}{(2 \pi)^{2+m}} \int_{B_{-} \backslash D} d \varphi\left(z_{1}, z_{2}\right) d \varphi\left(z_{2}, z_{1}\right) d \varphi\left(w, z_{2}\right)^{m}
$$

We first integrate over $w$. We get

$$
\int_{B_{-} \backslash D} d \varphi\left(w, z_{2}\right)^{m}=\varphi\left(z_{1}, z_{2}\right)^{m}-(2 \pi)^{m}\left[z_{1}, z_{2}\right]
$$

where

$$
\left[z_{1}, z_{2}\right]= \begin{cases}1 & z_{1} \text { is to the left of } z_{2} \\ 0 & \text { otherwise }\end{cases}
$$

Thus we must compute

$$
\frac{1}{(2 \pi)^{2+m}} \int \varphi\left(z_{1}, z_{2}\right)^{m} d \varphi\left(z_{1}, z_{2}\right) d \varphi\left(z_{2}, z_{1}\right)
$$




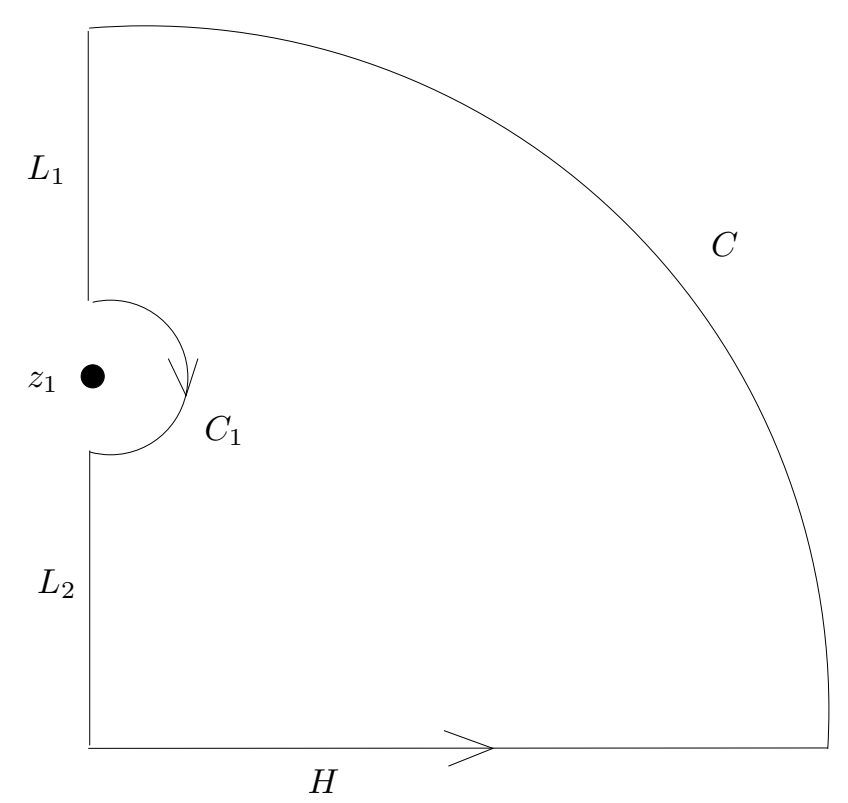

Figure 3

and

$$
\frac{1}{(2 \pi)^{2}} \int\left[z_{1}, z_{2}\right] d \varphi\left(z_{1}, z_{2}\right) d \varphi\left(z_{2}, z_{1}\right)
$$

Using Stokes theorem we rewrite (5.5) as a path integral as in Figure 3

$$
\frac{1}{(2 \pi)^{2}} \int_{L_{1} \cup C_{1} \cup L_{2} \cup H \cup C} \varphi\left(z_{1}, z\right) d \varphi\left(z, z_{1}\right)
$$

If $z \in L_{1,2} \cup H$ then $d \varphi\left(z, z_{1}\right)=0$. If $z \in C$ then $\varphi\left(z_{1}, z\right)=2 \pi$.

Thus

$$
\frac{1}{(2 \pi)^{2}} \int_{L_{1} \cup L_{2} \cup H \cup C} \varphi\left(z_{1}, z\right) d \varphi\left(z, z_{1}\right)=\frac{1}{(2 \pi)}(\pi-0)=\frac{1}{2}
$$

Now we compute

$$
\frac{1}{(2 \pi)^{2}} \int_{C_{1}} \varphi\left(z_{1}, z\right) d \varphi\left(z, z_{1}\right)
$$

We put $z=z_{1}+r e^{-i \theta}$ for $\left.\theta \in\right]-\frac{\pi}{2}, \frac{\pi}{2}\left[\right.$. Then $\varphi\left(z_{1}, z\right)=\frac{3}{2} \pi-\theta$ and hence $d \varphi\left(z, z_{1}\right)=-d \theta$.

Thus

$$
\begin{aligned}
\frac{1}{(2 \pi)^{2}} \int_{C_{1}} \varphi\left(z_{1}, z\right) d \varphi\left(z, z_{1}\right) & =-\frac{1}{(2 \pi)^{2}} \int_{-\pi / 2}^{\pi / 2}\left(\frac{3}{2} \pi-\theta\right) d \theta \\
& =\frac{1}{(2 \pi)^{2}} \int_{-\pi / 2}^{\pi / 2}\left(\theta-\frac{3}{2} \pi\right) d \theta \\
& =\frac{1}{(2 \pi)^{2}} \int_{-2 \pi}^{-\pi} \theta d \theta \\
& =-\frac{3}{8}
\end{aligned}
$$


and hence

$$
\frac{1}{(2 \pi)^{2}} \int\left[z_{1}, z_{2}\right] d \varphi\left(z_{1}, z_{2}\right) d \varphi\left(z_{2}, z_{1}\right)=\frac{1}{2}-\frac{3}{8}=\frac{1}{8}
$$

One the other hand by (4.3)

$$
\begin{aligned}
\frac{1}{(2 \pi)^{2+m}} \int \varphi\left(z_{1}, z_{2}\right)^{m} d \varphi\left(z_{1}, z_{2}\right) d \varphi\left(z_{2}, z_{1}\right) & =\frac{1}{(m+1)(2 \pi)^{2+m}} \int d \varphi\left(z_{1}, z_{2}\right)^{m+1} d \varphi\left(z_{2}, z_{1}\right) \\
& =\frac{1}{m+1}\left(\frac{1}{2}-\frac{1}{m+2}\right)
\end{aligned}
$$

so that our final formula is

$$
\beta_{2, m}=-\frac{1}{8}+\frac{1}{m+1}\left(\frac{1}{2}-\frac{1}{m+2}\right)
$$

5.2. Step 2. We claim

$$
\int_{z \in \mathbb{C} \backslash\left\{z_{1}, z_{2}\right\}} d \theta\left(z_{1}, z\right) d \theta\left(z, z_{2}\right)=0
$$

Since $d \theta\left(z, z_{2}\right)=d \theta\left(z_{2}, z\right)$ we may as well prove

$$
\int_{z \in \mathbb{C} \backslash\left\{z_{1}, z_{2}\right\}} d \theta\left(z_{1}, z\right) d \theta\left(z_{2}, z\right)=0
$$

We may assume $z_{1} \neq z_{2}$ since otherwise the claim is trivial. Consider the following figure

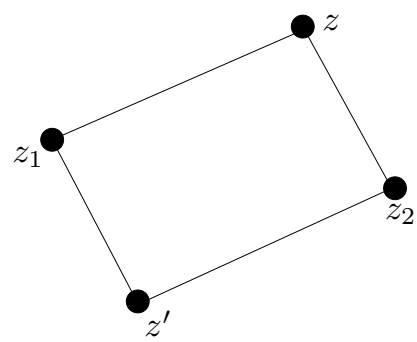

Then we have $d \theta\left(z_{1}, z\right) d \theta\left(z_{2}, z\right)=-d \theta\left(z_{1}, z^{\prime}\right) d \theta\left(z_{2}, z^{\prime}\right)$. Since the map $z \mapsto z^{\prime}$ is orientation preserving this proves what we want.

5.3. Step 3. Now we assume $n \geq 3$. First we observe

$$
\begin{aligned}
\beta_{n, m} & =(-1)^{n} \frac{1}{(2 \pi)^{2 n+m-2}} \int_{B_{-} \backslash D} d \varphi\left(w, z_{2}\right)^{m} d \varphi\left(z_{1}, z_{2}\right) \cdots d \varphi\left(z_{n-1}, z_{n}\right) d \varphi\left(z_{n}, z_{1}\right) d \varphi\left(w, z_{3}\right) \cdots d \varphi\left(w, z_{n}\right) \\
& =(-1)^{n} \frac{1}{(2 \pi)^{2 n+m-2}} \int_{\partial\left(B_{-} \backslash D\right)} \varphi\left(w, z_{2}\right)^{m} d \varphi\left(z_{1}, z_{2}\right) \cdots d \varphi\left(z_{n-1}, z_{n}\right) d \varphi\left(z_{n}, z_{1}\right) d \varphi\left(w, z_{3}\right) \cdots d \varphi\left(w, z_{n}\right)
\end{aligned}
$$

The components of $\partial\left(B_{-} \backslash D\right)$ which yield non-zero contributions to the integral have been listed in $\S 3.3$. Note that the contribution of (3.7) is zero by (5.6) (integrate first over $z_{i}$ ). We now list the contributing integrals with the correct signs.

(1) Consider the boundary component $\partial_{1}$ given by contracting all edges. The coordinates in the neighborhood of this component are given by $r, x_{2}^{\prime}, y_{2}^{\prime}, \ldots, x_{n}^{\prime}, y_{n}^{\prime}$

$$
\begin{aligned}
& y_{1}=1+r \\
& x_{i}=r x_{i}^{\prime} \\
& y_{i}=1+r y_{i}^{\prime}
\end{aligned}
$$


with $r \geq 0$. In these coordinate the orientation form on $B_{-}$is (up to a positive multiple) given by

$$
-d r d x_{2}^{\prime} d y_{2}^{\prime} \cdots d x_{n}^{\prime} d y_{n}^{\prime}
$$

and hence $\partial_{1}$ is oriented by $d x_{2}^{\prime} d y_{2}^{\prime} \cdots d x_{n}^{\prime} d y_{n}^{\prime}$. Whence the contribution to the integral is equal to $(-1)^{n} \epsilon_{n, m}$.

(2) Now consider the boundary component $\partial_{2}$ given by contracting $\left\{z_{1}, w\right\}$. We claim $\partial_{2} \cong C_{n, 0}$, taking orientations into account. Coordinates in a neighborhood of $\partial_{2}$ are given by $r, x_{2}, y_{2}, \ldots, x_{n}, y_{n}$ where $y_{1}=1+r$ and $r \geq 0$. Thus the orientation form is $-d r d x_{2} d y_{2} \cdots x_{n}, y_{n}$ and hence $\partial_{2}$ is oriented by $d x_{2} d y_{2} \cdots x_{n}, y_{n}$ which is the normal orientation on $C_{n, 0}$ [1]. We obtain

$$
\begin{aligned}
& (-1)^{n} \frac{1}{(2 \pi)^{2 n+m-2}} \int_{\partial_{2}} \varphi\left(w, z_{2}\right)^{m} d \varphi\left(z_{1}, z_{2}\right) \cdots d \varphi\left(z_{n-1}, z_{n}\right) d \varphi\left(z_{n}, z_{1}\right) d \varphi\left(w, z_{3}\right) \cdots d \varphi\left(w, z_{n}\right) \\
& =(-1)^{n} \frac{1}{(2 \pi)^{2 n+m-2}} \int_{C_{n, 0}} \varphi\left(w, z_{2}\right)^{m} d \varphi\left(w, z_{2}\right) \cdots d \varphi\left(z_{n-1}, z_{n}\right) d \varphi\left(z_{n}, z_{1}\right) d \varphi\left(w, z_{3}\right) \cdots d \varphi\left(w, z_{n}\right) \\
& =(-1)^{n} \frac{1}{(m+1)(2 \pi)^{2 n+m-2}} \int_{C_{n, 0}} d \varphi\left(w, z_{2}\right)^{m+1} d \varphi\left(z_{2}, z_{3}\right) \cdots d \varphi\left(z_{n-1}, z_{n}\right) d \varphi\left(z_{n}, z_{1}\right) d \varphi\left(w, z_{3}\right) \cdots d \varphi\left(w, z_{n}\right) \\
& =-\frac{1}{(m+1)(2 \pi)^{2 n+m-2}} \int_{C_{n, 0}} d \varphi\left(z_{2}, z_{3}\right) \cdots d \varphi\left(z_{n-1}, z_{n}\right) d \varphi\left(z_{n}, z_{1}\right) d \varphi\left(w, z_{2}\right)^{m+1} d \varphi\left(w, z_{3}\right) \cdots d \varphi\left(w, z_{n}\right) \\
& =-\frac{1}{m+1} \alpha_{n-1, m+1}
\end{aligned}
$$

(3) Let $\partial_{3}$ be obtained by contracting $\left(z_{1}, z_{2}\right)$. We claim that $\partial_{3}=-C_{2}^{\prime} \times B_{-}^{\prime}$ where $B_{-}^{\prime}$ is the analogue of $B_{-}$inside $C_{n, 0}$ and where $C_{2}^{\prime}$ is the part of $C_{2}$ where the two points are not on the same vertical line. Thus $C_{2}^{\prime}$ and hence $\partial_{3}$ has two connected components.

Coordinates in a neighborhood of $\partial_{3}$ are given by $r, \theta, y_{1}, x_{3}, y_{3}, \ldots, x_{n}, y_{n}$ where

$$
\begin{aligned}
& x_{2}=r \cos \theta \\
& y_{2}=y_{1}+r \sin \theta
\end{aligned}
$$

and $r \geq 0$ and $\theta \notin\{\pi / 2,-\pi / 2\}$ (the latter because of the branch cut involving $\left.\varphi\left(w, z_{2}\right)\right)$. Hence we find that the orientation form on $B_{-}$is up to a positive factor given by

$$
-d y_{1} d r d \theta d x_{3} d y_{3} \cdots d x_{n} d y_{n}
$$

Therefore the orientation form on $\partial_{3}$ (using the outward normal) is given by

$$
-d y_{1} d \theta d x_{3} d y_{2} \cdots d x_{n} d y_{n}=(-d \theta) \times\left(-d y_{1} d x_{3} d y_{3} \cdots d x_{n} d y_{n}\right)
$$

which does indeed represent the orientation on $-C_{2}^{\prime} \times B_{-}^{\prime}$. 
THE KONTSEVICH WEIGHT OF A WHEEL WITH SPOKES POINTING OUTWARD 21

The resulting integral is now

$$
\begin{aligned}
& (-1)^{n} \frac{1}{(2 \pi)^{2 n+m-2}} \int_{\partial_{3}} \varphi\left(w, z_{2}\right)^{m} d \varphi\left(z_{1}, z_{2}\right) \cdots d \varphi\left(z_{n-1}, z_{n}\right) d \varphi\left(z_{n}, z_{1}\right) d \varphi\left(w, z_{3}\right) \cdots d \varphi\left(w, z_{n}\right) \\
& =(-1)^{n+1} \frac{1}{2} \frac{1}{(2 \pi)^{2 n-1}} \int_{B_{-}^{\prime}} d \varphi\left(z_{2}, z_{3}\right) \cdots d \varphi\left(z_{n-1}, z_{n}\right) d \varphi\left(z_{n}, z_{2}\right) d \varphi\left(w, z_{3}\right) \cdots d \varphi\left(w, z_{n}\right) \\
& =(-1)^{n+1} \frac{1}{2} \beta_{n-1,1}
\end{aligned}
$$

where the factor $1 / 2$ comes from the fact that $\varphi\left(w, z_{2}\right)=0$ if $z_{2}$ is to the left of $z_{1}$.

(4) Let $\partial_{4}$ be obtained by contracting $\left(z_{2}, z_{3}\right)$. A similar computation as above shows $\partial_{4} \cong-C_{2} \times B_{-}^{\prime}$. The resulting integral is

$$
\begin{aligned}
& (-1)^{n} \frac{1}{(2 \pi)^{2 n+m-2}} \int_{\partial_{4}} \varphi\left(w, z_{2}\right)^{m} d \varphi\left(z_{1}, z_{2}\right) \cdots d \varphi\left(z_{n-1}, z_{n}\right) d \varphi\left(z_{n}, z_{1}\right) d \varphi\left(w, z_{3}\right) \cdots d \varphi\left(w, z_{n}\right) \\
& =(-1)^{n+1} \frac{1}{(2 \pi)^{2 n+m-2}} \int_{\partial_{4}} \varphi\left(w, z_{2}\right)^{m} d \varphi\left(z_{2}, z_{3}\right) d \varphi\left(z_{1}, z_{2}\right) d \varphi\left(z_{3}, z_{4}\right) \cdots d \varphi\left(z_{n-1}, z_{n}\right) \times \\
& =(-1)^{n} \frac{1}{(2 \pi)^{2 n+m-3}} \int_{B_{-}^{\prime}} \varphi\left(w, z_{3}\right)^{m} d \varphi\left(z_{1}, z_{3}\right) d \varphi\left(z_{3}, z_{4}\right) \cdots d \varphi\left(z_{n-1}, z_{n}\right) \times \\
& =(-1)^{n} \frac{1}{(m+1)(2 \pi)^{2 n+m-3}} \int_{B_{-}^{\prime}} d \varphi\left(z_{1}, z_{3}\right) d \varphi\left(z_{3}, z_{4}\right) \cdots d \varphi\left(z_{n-1}, z_{n}\right) \times \\
& \left.=(-1)^{n} \frac{1}{m+1} \beta_{n-1, m+1}\right) d \varphi\left(w, z_{3}\right) \cdots d \varphi\left(w, z_{n}\right)
\end{aligned}
$$

Combining all contributions we get (5.3).

\section{A RECURSiOn RELATiON FOR $\bar{\beta}_{n, m}$}

By our definition in $\S 3.4, \bar{\beta}_{n, m}$ is the integral associated to the enhanced graph

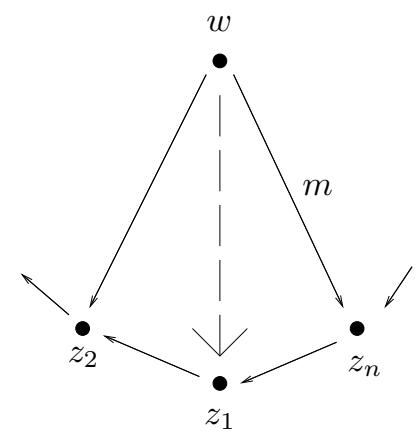

Thus with our standard edge ordering

$\bar{\beta}_{n, m}=\frac{1}{(2 \pi)^{2 n+m-2}} \int_{B_{-} \backslash D} d \varphi\left(z_{1}, z_{2}\right) \cdots d \varphi\left(z_{n-1}, z_{n}\right) d \varphi\left(z_{n}, z_{1}\right) d \varphi\left(w, z_{2}\right) \cdots d \varphi\left(w, z_{n}\right)^{m}$

where

$$
B_{-}=\left\{\operatorname{Re} z_{1}=\operatorname{Re} w+, \operatorname{Im} z_{1}>\operatorname{Im} w\right\} \subset C_{n+1,0}
$$




$$
D=\left\{\operatorname{Re} z_{n}=\operatorname{Re} w, \operatorname{Im} z_{2}>\operatorname{Im} w\right\} \subset C_{n+1,0}
$$

and where $B_{-}$is oriented with the outgoing normal (see (5.1)). Clearly we have

$$
\begin{gathered}
\bar{\beta}_{2, m}=\beta_{2, m} \\
\bar{\beta}_{n, 1}=\beta_{n, 1}
\end{gathered}
$$

In this section we will prove for $n \geq 3$

$$
\bar{\beta}_{n, m}=(-1)^{n} \frac{1}{2} \beta_{n-1,1}+(-1)^{n+1} \frac{1}{m+1} \bar{\beta}_{n-1, m+1}+\bar{\epsilon}_{n, m}
$$

where $\bar{\epsilon}_{n, m}$ is the integral associated to the graph

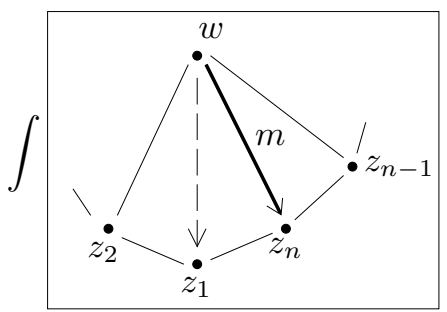

(see §3.4). Thus with our standard orientation on edges

$\bar{\epsilon}_{n, m}=\frac{1}{(2 \pi)^{2 n+m-2}} \int \theta\left(w, z_{n}\right)^{m} d \theta\left(z_{1}, z_{2}\right) d \theta\left(z_{2}, z_{3}\right) \cdots d \theta\left(z_{n}, z_{1}\right) d \theta\left(w, z_{2}\right) \cdots d \theta\left(w, z_{n-1}\right)$

Here we put $w=0, z_{1}=i$ and the integral is over the complement of the diagonal in $(\mathbb{C}-\{0, i\})^{n-1}$.

6.1. Proof. We assume $n \geq 3$. We first observe

$$
\begin{aligned}
\bar{\beta}_{n, m} & =\frac{1}{(2 \pi)^{2 n+m-2}} \int_{B_{-} \backslash D} d \varphi\left(w, z_{n}\right)^{m} d \varphi\left(z_{1}, z_{2}\right) \cdots d \varphi\left(z_{n-1}, z_{n}\right) d \varphi\left(z_{n}, z_{1}\right) d \varphi\left(w, z_{2}\right) \cdots d \varphi\left(w, z_{n-1}\right) \\
& =\frac{1}{(2 \pi)^{2 n+m-2}} \int_{\partial\left(B_{-} \backslash D\right)} \varphi\left(w, z_{n}\right)^{m} d \varphi\left(z_{1}, z_{2}\right) \cdots d \varphi\left(z_{n-1}, z_{n}\right) d \varphi\left(z_{n}, z_{1}\right) d \varphi\left(w, z_{2}\right) \cdots d \varphi\left(w, z_{n-1}\right)
\end{aligned}
$$

The components of $\partial\left(B_{-} \backslash D\right)$ which yield non-zero contributions to the integral have been listed in $\S 3.3$. Note that the contribution of (3.8) is zero by (5.6) (integrate first over $\left.z_{n-1}\right)$. We now list the contributing integrals with the correct signs.

(1) Consider the boundary component $\partial_{1}$ given by contracting all edges. Then a computation similar to $\S 5.3(1)$ yields that the contribution is $\bar{\epsilon}_{n, m}$.

(2) Now consider the boundary component $\partial_{2}$ given by contracting $\left(z_{n-1}, w\right)$. It this case we get an integral which contains as a subintegral (up to a scalar factor)

$$
\int_{z_{n} \in \mathcal{H} \backslash\left\{w, z_{1}\right\}} d \varphi\left(w, z_{n}\right)^{m+1} d \varphi\left(z_{n}, z_{1}\right)
$$

Using (4.3) this is equal to

$$
(2 \pi)^{m+1} \varphi\left(w, z_{1}\right)-2 \pi \varphi\left(w, z_{1}\right)^{m+1}=(2 \pi)^{m+1} \times 2 \pi-2 \pi \times(2 \pi)^{m+1}=0
$$

So we are lucky that there is no contribution in this case. 
THE KONTSEVICH WEIGHT OF A WHEEL WITH SPOKES POINTING OUTWARD 23

(3) Let $\partial_{3}$ be obtained by contracting $\left(z_{1}, z_{n}\right)$. A similar computation as in $\S 5.33)$ shows $\partial_{3}=-C_{2}^{\prime} \times B_{-}^{\prime}$. The resulting integral is

$$
\begin{aligned}
& \frac{1}{(2 \pi)^{2 n+m-2}} \int_{\partial_{3}} \varphi\left(w, z_{n}\right)^{m} d \varphi\left(z_{1}, z_{2}\right) \cdots d \varphi\left(z_{n-1}, z_{n}\right) d \varphi\left(z_{n}, z_{1}\right) d \varphi\left(w, z_{2}\right) \cdots d \varphi\left(w, z_{n-1}\right) \\
& =(-1)^{n-1} \frac{1}{(2 \pi)^{2 n+m-2}} \int_{\partial_{3}} \varphi\left(w, z_{n}\right)^{m} d \varphi\left(z_{n}, z_{1}\right) d \varphi\left(z_{1}, z_{2}\right) \cdots d \varphi\left(z_{n-1}, z_{n}\right) d \varphi\left(w, z_{2}\right) \cdots d \varphi\left(w, z_{n-1}\right) \\
& =(-1)^{n} \frac{1}{2} \frac{1}{(2 \pi)^{2 n-2}} \int_{B_{-}^{\prime}} d \varphi\left(z_{1}, z_{2}\right) \cdots d \varphi\left(z_{n-1}, z_{1}\right) d \varphi\left(w, z_{2}\right) \cdots d \varphi\left(w, z_{n-1}\right) \\
& =(-1)^{n} \frac{1}{2} \bar{\beta}_{n-1,1}=(-1)^{n} \frac{1}{2} \beta_{n-1,1}
\end{aligned}
$$

where the factor $1 / 2$ comes from the fact that $\varphi\left(w, z_{n}\right)=0$ if $z_{2}$ is to the left of $z_{1}$.

(4) Let $\partial_{4}$ be obtained by contracting $\left(z_{n-1}, z_{n}\right)$. A similar computation as in $\S 5.33)$ shows $\partial_{4} \cong-C_{2} \times B_{-}^{\prime}$. The resulting integral is

$$
\begin{aligned}
& \frac{1}{(2 \pi)^{2 n+m-2}} \int_{\partial_{4}} \varphi\left(w, z_{n}\right)^{m} d \varphi\left(z_{1}, z_{2}\right) \cdots d \varphi\left(z_{n-1}, z_{n}\right) d \varphi\left(z_{n}, z_{1}\right) d \varphi\left(w, z_{2}\right) \cdots d \varphi\left(w, z_{n-1}\right) \\
& =(-1)^{n} \frac{1}{(2 \pi)^{2 n+m-2}} \int_{\partial_{4}} \varphi\left(w, z_{n}\right)^{m} d \varphi\left(z_{n-1}, z_{n}\right) d \varphi\left(z_{1}, z_{2}\right) \cdots d \varphi\left(z_{n-2}, z_{n-1}\right) \times \\
& =(-1)^{n+1} \frac{1}{(2 \pi)^{2 n+m-3}} \int_{B_{-}^{\prime}} \varphi\left(w, z_{n-1}\right)^{m} d \varphi\left(z_{1}, z_{2}\right) \cdots d \varphi\left(z_{n-2}, z_{n-1}\right) \times \\
& =(-1)^{n+1} \frac{d \varphi\left(z_{n-1}, z_{1}\right) d \varphi\left(w, z_{2}\right) \cdots d \varphi\left(w, z_{n-1}\right)}{(m+1)(2 \pi)^{2 n+m-3}} \int_{B_{-}^{\prime}} d \varphi\left(z_{1}, z_{2}\right) \cdots d \varphi\left(z_{n-2}, z_{n-1}\right) \times \\
& =(-1)^{n+1} \frac{1}{m+1} \bar{\beta}_{n-1, m+1}
\end{aligned}
$$

Combining all contributions we get (6.1).

\section{A RECURSiOn NOT INVOLVING UNKNOWN QUANTITIES.}

Put

$$
\hat{\beta}_{n, m}=\frac{1}{2}\left(\beta_{n, m}+(-1)^{n} \bar{\beta}_{n, m}\right)
$$

We claim

$$
\hat{\beta}_{n, m}=-\frac{1}{2(m+1)} \alpha_{n-1, m+1}+(-1)^{n+1} \frac{1}{2} \hat{\beta}_{n-1,1}+(-1)^{n} \frac{1}{m+1} \hat{\beta}_{n-1, m+1}
$$

with initial condition

$$
\hat{\beta}_{2, m}=-\frac{1}{8}+\frac{1}{m+1}\left(\frac{1}{2}-\frac{1}{m+2}\right)
$$


7.1. Step 1. We claim first that

$$
\epsilon_{n, m}=-\bar{\epsilon}_{n, m}
$$

We have

$\epsilon_{n, m}=\frac{1}{(2 \pi)^{2 n+m-2}} \int \theta\left(w, z_{2}\right)^{m} d \theta\left(z_{1}, z_{2}\right) d \theta\left(z_{2}, z_{3}\right) \cdots d \theta\left(z_{n}, z_{1}\right) d \theta\left(w, z_{3}\right) \cdots d \theta\left(w, z_{n}\right)$

Here we put $w=0, z_{1}=i$ and the integral is over a suitable open subset of $\mathbb{C}^{2 n-1}$.

We apply the permutation $(2 n)(3 n-1) \cdots$ to $z_{1}, \ldots, z_{n}$. This does not change the orientation. We find

$$
\begin{aligned}
\epsilon_{n, m} & =\frac{1}{(2 \pi)^{2 n+m-2}} \int \theta\left(w, z_{n}\right)^{m} d \theta\left(z_{1}, z_{n}\right) d \theta\left(z_{n}, z_{n-1}\right) \cdots d \theta\left(z_{2}, z_{1}\right) d \theta\left(w, z_{n-1}\right) \cdots d \theta\left(w, z_{2}\right) \\
& =\frac{1}{(2 \pi)^{2 n+m-2}} \int \theta\left(w, z_{n}\right)^{m} d \theta\left(z_{n}, z_{1}\right) d \theta\left(z_{n-1}, z_{n}\right) \cdots d \theta\left(z_{1}, z_{2}\right) d \theta\left(w, z_{n-1}\right) \cdots d \theta\left(w, z_{2}\right) \\
& =(-1)^{s} \bar{\epsilon}_{n, m}
\end{aligned}
$$

The sign $(-1)^{s}$ comes from the fact that we have invert the order of the 1-forms in $d \theta\left(z_{n}, z_{1}\right) d \theta\left(z_{n-1} z_{n}\right) \cdots d \theta\left(z_{1}, z_{2}\right)$ and in $d \theta\left(w, z_{n-1}\right) \cdots d \theta\left(w, z_{2}\right)$. Thus

$$
s=\frac{n(n-1)}{2}+\frac{(n-2)(n-3)}{2}=n^{2}-3 n+3
$$

which is always an odd number. This proves (7.3).

7.2. Step 2. Formula (7.2) is obvious from (5.2) and the fact that $\hat{\beta}_{2, m}=\beta_{2, m}$. Formula (7.1) follows from (5.3) and (6.1) together with the following computation

$$
\begin{aligned}
\hat{\beta}_{n, m} & =\frac{1}{2}\left(-\frac{1}{m+1} \alpha_{n-1, m+1}+(-1)^{n+1} \frac{1}{2} \beta_{n-1,1}+\frac{1}{2} \beta_{n-1,1}+(-1)^{n} \frac{1}{m+1} \beta_{n-1, m+1}-\frac{1}{m+1} \bar{\beta}_{n-1, m+1}\right) \\
& =-\frac{1}{2(m+1)} \alpha_{n-1, m+1}+(-1)^{n+1} \frac{1}{2} \hat{\beta}_{n-1,1}+(-1)^{n} \frac{1}{m+1} \hat{\beta}_{n-1, m+1}
\end{aligned}
$$

\section{A FORMUla FOR $w_{n}$}

In this section we prove the following fact

$$
w_{n}= \begin{cases}\hat{\beta}_{n, 1}-\frac{1}{2} \alpha_{n-1,2} & \text { if } n \text { is even } \\ 0 & \text { if } n \text { is odd }\end{cases}
$$

8.1. Step 1. We assume $n$ odd. Put $w=i$. We identify $C_{n, 0}$ with an open subset of $\mathcal{H}^{n}$. The orientation is derived from the standard orientation on $\mathcal{H}^{n}$ (according to [1]).

We consider the map $\alpha: \mathcal{H} \rightarrow \mathcal{H}: x+i y \mapsto-x+i y$ and we extend $\alpha$ diagonally to a map $\mathcal{H}^{n} \rightarrow \mathcal{H}^{n}$ also denoted by $\alpha$. $\alpha$ multiplies the orientation by $(-1)^{n}$.

Hence we have

$w_{n}=(-1)^{n} \frac{1}{(2 \pi)^{2 n}} \int_{C_{n, 0}} d \varphi\left(\alpha\left(z_{1}\right), \alpha\left(z_{2}\right)\right) \cdots d \varphi\left(\alpha\left(z_{1}\right), \alpha\left(z_{n}\right)\right) d \varphi\left(w, \alpha\left(z_{1}\right)\right) \cdots d \varphi\left(w, \alpha\left(z_{n}\right)\right)$

(since $\alpha(w)=w)$.

It is now easy to see that $\varphi(\alpha(u), \alpha(v))=2 \pi-\varphi(u, v)$ and hence $d \varphi(\alpha(u), \alpha(v))=$ $-d \varphi(u, v)$. Thus

$$
w_{n}=(-1)^{n}(-1)^{2 n} w_{n}=(-1)^{n} w_{n}
$$

Since $n$ is odd this implies $w_{n}=0$. 
8.2. Step 2. Consider the following enhanced graph

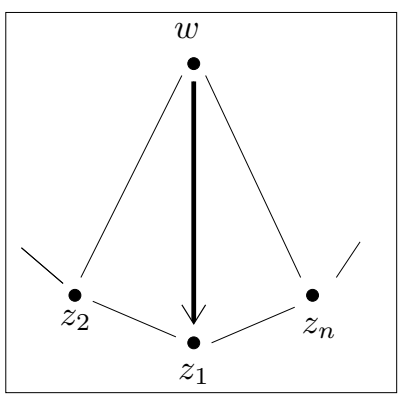

We claim that its corresponding integral

$$
\delta=\frac{1}{(2 \pi)^{2 n}} \int_{C_{n+1}} \theta\left(w, z_{1}\right) d \theta\left(z_{1}, z_{2}\right) \cdots d \theta\left(z_{n}, z_{1}\right) d \theta\left(w, z_{2}\right) \cdots d \theta\left(w, z_{n}\right)
$$

is zero when $n$ is even.

It's easy to see that permuting the points does not change the orientation on $C_{n+1}$. We apply the permutation $(2 n)(3 n-1) \cdots$ for $z_{1}, z_{2}, \ldots, z_{n}$. Hence we also have

$$
\begin{aligned}
\delta & =\frac{1}{(2 \pi)^{2 n}} \int_{C_{n+1}} \theta\left(w, z_{1}\right) d \theta\left(z_{1}, z_{n}\right) d \theta\left(z_{n} z_{n-1}\right) \cdots d \theta\left(z_{2}, z_{1}\right) d \theta\left(w, z_{n}\right) \cdots d \theta\left(w, z_{2}\right) \\
& =\frac{1}{(2 \pi)^{2 n}} \int_{C_{n+1}} \theta\left(w, z_{1}\right) d \theta\left(z_{n}, z_{1}\right) d \theta\left(z_{n-1} z_{n}\right) \cdots d \theta\left(z_{1}, z_{2}\right) d \theta\left(w, z_{n}\right) \cdots d \theta\left(w, z_{2}\right)
\end{aligned}
$$

To put this back in standard form we have to invert the order of the 1-forms in $d \theta\left(z_{n}, z_{1}\right) d \theta\left(z_{n-1} z_{n}\right) \cdots d \theta\left(z_{1}, z_{2}\right)$ and in $d \theta\left(w, z_{n}\right) \cdots d \theta\left(w, z_{2}\right)$. This introduces a cumulative sign of $(-1)^{s}$ where

$$
s=\frac{n(n-1)}{2}+\frac{(n-1)(n-2)}{2}=(n-1)^{2}
$$

Thus

$$
\delta=-(-1)^{n} \delta
$$

and hence if $n$ is even then $\delta=0$.

8.3. Step 3. Now we assume $n$ even. As a pedagogical device we keep signs of the form $(-1)^{n}$ below. We first observe

$$
\begin{aligned}
w_{n} & =(-1)^{n} \frac{1}{(2 \pi)^{2 n}} \int_{C_{n+1,0}} d \varphi\left(w, z_{1}\right) d \varphi\left(z_{1}, z_{2}\right) \cdots d \varphi\left(z_{n-1}, z_{n}\right) d \varphi\left(z_{n}, z_{1}\right) d \varphi\left(w, z_{2}\right) \cdots d \varphi\left(w, z_{n}\right) \\
& =(-1)^{n} \frac{1}{(2 \pi)^{2 n}} \int_{\text {boundary }} \varphi\left(w, z_{1}\right) d \varphi\left(z_{1}, z_{2}\right) \cdots d \varphi\left(z_{n-1}, z_{n}\right) d \varphi\left(z_{n}, z_{1}\right) d \varphi\left(w, z_{2}\right) \cdots d \varphi\left(w, z_{n}\right)
\end{aligned}
$$

The boundary consists of two parts. One part consists of both sides of the branch cut $B_{ \pm}$.

$$
\begin{aligned}
& B_{-}=\left\{\operatorname{Re} z_{1}=\operatorname{Re} w+, \operatorname{Im} z_{1}>\operatorname{Im} w\right\} \subset C_{n+1,0} \\
& B_{+}=\left\{\operatorname{Re} z_{1}=\operatorname{Re} w-, \operatorname{Im} z_{1}>\operatorname{Im} w\right\} \subset C_{n+1,0}
\end{aligned}
$$

The values of $\varphi(w,-)$ on both sides of the branch cut differ by $2 \pi$. Hence the contribution of this part of the boundary is equal to

$$
(-1)^{n} \frac{1}{(2 \pi)^{2 n-1}} \int_{B_{-}} \varphi\left(w, z_{1}\right) d \varphi\left(z_{1}, z_{2}\right) \cdots d \varphi\left(z_{n-1}, z_{n}\right) d \varphi\left(z_{n}, z_{1}\right) d \varphi\left(w, z_{2}\right) \cdots d \varphi\left(w, z_{n}\right)
$$


which is nothing but

$$
(-1)^{n} \beta_{n, 1}
$$

In $\S 3.2$ we have listed the "classical" parts of the boundary which contribute to the integral. Here we give the contributions with the precise signs. Note that the contribution of (8.2) is zero by Step 2 .

(1) Let $\partial_{1}$ be the boundary component given by contracting $\left(w, z_{n}\right)$. According to [1] we have $\partial_{1} \cong-C_{2} \times C_{n, 0}$. The contribution to the integral is

$$
\begin{aligned}
& (-1)^{n} \frac{1}{(2 \pi)^{2 n}} \int_{\partial_{1}} \varphi\left(w, z_{1}\right) d \varphi\left(z_{1}, z_{2}\right) \cdots d \varphi\left(z_{n-1}, z_{n}\right) d \varphi\left(z_{n}, z_{1}\right) d \varphi\left(w, z_{2}\right) \cdots d \varphi\left(w, z_{n-1}\right) d \varphi\left(w, z_{n}\right) \\
= & (-1)^{n} \frac{1}{(2 \pi)^{2 n}} \int_{\partial_{1}} \varphi\left(w, z_{1}\right) d \varphi\left(w, z_{n}\right) d \varphi\left(z_{1}, z_{2}\right) \cdots d \varphi\left(z_{n-1}, z_{n}\right) d \varphi\left(z_{n}, z_{1}\right) d \varphi\left(w, z_{2}\right) \cdots d \varphi\left(w, z_{n-1}\right) \\
= & (-1)^{n+1} \frac{1}{(2 \pi)^{2 n-1}} \int_{C_{n, 0}} \varphi\left(w, z_{1}\right) d \varphi\left(z_{1}, z_{2}\right) \cdots d \varphi\left(z_{n-1}, w\right) d \varphi\left(w, z_{1}\right) d \varphi\left(w, z_{2}\right) \cdots d \varphi\left(w, z_{n-1}\right) \\
= & (-1)^{n+1} \frac{1}{2(2 \pi)^{2 n-1}} \int_{C_{n, 0}} d \varphi\left(z_{1}, z_{2}\right) \cdots d \varphi\left(z_{n-1}, w\right) d \varphi\left(w, z_{1}\right)^{2} d \varphi\left(w, z_{2}\right) \cdots d \varphi\left(w, z_{n-1}\right) \\
= & (-1)^{n+1} \frac{1}{2} \alpha_{n-1,2}
\end{aligned}
$$

(2) Let $\partial_{2} \cong-C_{2} \times C_{n, 0}$ be obtained by contracting $\left(z_{1}, z_{2}\right)$. We obtain

$$
\begin{aligned}
& (-1)^{n} \frac{1}{(2 \pi)^{2 n}} \int_{\partial_{2}} \varphi\left(w, z_{1}\right) d \varphi\left(z_{1}, z_{2}\right) \cdots d \varphi\left(z_{n-1}, z_{n}\right) d \varphi\left(z_{n}, z_{1}\right) d \varphi\left(w, z_{2}\right) \cdots d \varphi\left(w, z_{n}\right) \\
= & (-1)^{n+1} \frac{1}{(2 \pi)^{2 n-1}} \int_{C_{n, 0}} \varphi\left(w, z_{2}\right) d \varphi\left(z_{2}, z_{3}\right) \cdots d \varphi\left(z_{n-1}, z_{n}\right) d \varphi\left(z_{n}, z_{2}\right) d \varphi\left(w, z_{2}\right) \cdots d \varphi\left(w, z_{n}\right) \\
= & (-1)^{n+1} \frac{1}{(2)(2 \pi)^{2 n-1}} \int_{C_{n, 0}} d \varphi\left(z_{2}, z_{3}\right) \cdots d \varphi\left(z_{n-1}, z_{n}\right) d \varphi\left(z_{n}, z_{2}\right) d \varphi\left(w, z_{2}\right)^{2} \cdots d \varphi\left(w, z_{n}\right)
\end{aligned}
$$

(3) Let $\partial_{3} \cong-C_{2} \times C_{n, 0}$ be obtained by contracting $\left(z_{n}, z_{1}\right)$. We obtain

$$
\begin{aligned}
& (-1)^{n} \frac{1}{(2 \pi)^{2 n}} \int_{\partial_{3}} \varphi\left(w, z_{1}\right) d \varphi\left(z_{1}, z_{2}\right) \cdots d \varphi\left(z_{n-1}, z_{n}\right) d \varphi\left(z_{n}, z_{1}\right) d \varphi\left(w, z_{2}\right) \cdots d \varphi\left(w, z_{n}\right) \\
= & -\frac{1}{(2 \pi)^{2 n}} \int_{\partial_{3}} \varphi\left(w, z_{1}\right) d \varphi\left(z_{n}, z_{1}\right) d \varphi\left(z_{1}, z_{2}\right) \cdots d \varphi\left(z_{n-1}, z_{n}\right) d \varphi\left(w, z_{2}\right) \cdots d \varphi\left(w, z_{n}\right) \\
= & \frac{1}{(2 \pi)^{2 n-1}} \int_{C_{n, 0}} \varphi\left(w, z_{1}\right) d \varphi\left(z_{1}, z_{2}\right) \cdots d \varphi\left(z_{n-1}, z_{1}\right) d \varphi\left(w, z_{2}\right) \cdots d \varphi\left(w, z_{n-1}\right) d \varphi\left(w, z_{1}\right) \\
= & (-1)^{n-2} \frac{1}{(2 \pi)^{2 n-1}} \int_{C_{n, 0}} \varphi\left(w, z_{1}\right) d \varphi\left(z_{1}, z_{2}\right) \cdots d \varphi\left(z_{n-1}, z_{1}\right) d \varphi\left(w, z_{1}\right) d \varphi\left(w, z_{2}\right) \cdots d \varphi\left(w, z_{n-1}\right) \\
= & (-1)^{n-2} \frac{1}{(2)(2 \pi)^{2 n-1}} \int_{C_{n, 0}} d \varphi\left(z_{1}, z_{2}\right) \cdots d \varphi\left(z_{n-1}, z_{1}\right) d \varphi\left(w, z_{1}\right)^{2} d \varphi\left(w, z_{2}\right) \cdots d \varphi\left(w, z_{n-1}\right)
\end{aligned}
$$

Hence the contributions of $\partial_{2}$ and $\partial_{3}$ cancel. The contributions of $\partial_{1}$ and $B_{-}$yield (8.1) in case $n$ is even.

\section{SOLVING THE RECURSION}


9.1. The problem. For the benefit of the reader we restate the recursion relations we have derived. See $(4.1)(4.2)(7.1)(7.2)(8.1)$.

$$
\begin{aligned}
\alpha_{0, m} & =1 \\
\alpha_{n, m} & =(-1)^{n}\left(\frac{1}{2} \alpha_{n-1,2}-\frac{1}{m+1} \alpha_{n-1, m+1}\right) \quad(\text { for } n \geq 1) \\
\hat{\beta}_{2, m} & =-\frac{1}{8}+\frac{1}{m+1}\left(\frac{1}{2}-\frac{1}{m+2}\right) \\
\hat{\beta}_{n, m} & =-\frac{1}{2(m+1)} \alpha_{n-1, m+1}+(-1)^{n+1} \frac{1}{2} \hat{\beta}_{n-1,1}+(-1)^{n} \frac{1}{m+1} \hat{\beta}_{n-1, m+1} \quad(\text { for } n \geq 3) \\
w_{n} & =\hat{\beta}_{n, 1}-\frac{1}{2} \alpha_{n-1,2} \quad(\text { for } n \geq 2 \text { even })
\end{aligned}
$$

9.2. Eliminating some signs and fractions. We simplify the equations by putting

$$
\tilde{\alpha}_{n, m}=(-1)^{\frac{n(n+1)}{2}} \frac{1}{m !} \alpha_{n, m}
$$

Then we have

and

$$
\tilde{\alpha}_{0, m}=\frac{1}{m !}
$$

$$
\tilde{\alpha}_{n, m}=\frac{1}{m !} \tilde{\alpha}_{n-1,2}-\tilde{\alpha}_{n-1, m+1}
$$

Similarly we put

$$
\tilde{\beta}_{n, m}=(-1)^{\frac{n(n+1)}{2}} \frac{\hat{\beta}_{n, m}}{m !}
$$

so that we get.

$$
\tilde{\beta}_{2, m}=\frac{1}{8 m !}-\frac{1}{2(m+1) !}+\frac{1}{(m+2) !}
$$

and

$$
\tilde{\beta}_{n, m}=-(-1)^{n} \frac{1}{2} \tilde{\alpha}_{n-1, m+1}-\frac{1}{2 m !} \tilde{\beta}_{n-1,1}+\tilde{\beta}_{n-1, m+1}
$$

9.3. Computing $\tilde{\alpha}$. Iterating (9.1) we find

$$
\begin{aligned}
\tilde{\alpha}_{n, 2} & =\frac{1}{2 !} \tilde{\alpha}_{n-1,2}-\tilde{\alpha}_{n-1,3} \\
& =\frac{1}{2 !} \tilde{\alpha}_{n-1,2}-\frac{1}{3 !} \tilde{\alpha}_{n-2,2}+\tilde{\alpha}_{n-2,4} \\
& =\left(\frac{1}{2 !} \tilde{\alpha}_{n-1,2}-\frac{1}{3 !} \tilde{\alpha}_{n-2,2}+\cdots+(-1)^{n+1} \frac{1}{(n+1) !} \tilde{\alpha}_{0,2}\right)+(-1)^{n} \tilde{\alpha}_{0, n+2}
\end{aligned}
$$

Thus if we put

$$
A_{2}=\sum_{n \geq 0} \tilde{\alpha}_{n, 2} x^{n}
$$

we get

$$
A_{2}=\left(\frac{x}{2 !}-\frac{x^{2}}{3 !}+\cdots\right) A_{2}+\sum_{n \geq 0}(-1)^{n} \frac{x^{n}}{(n+2) !}
$$

Here

$$
\frac{x}{2 !}-\frac{x^{2}}{3 !}+\cdots=\frac{e^{-x}-1+x}{x}
$$


and

from which we deduce

$$
\sum_{n \geq 0}(-1)^{n} \frac{x^{n}}{(n+2) !}=\frac{e^{-x}-1+x}{x^{2}}
$$

For use below we record

$$
A_{2}=-\frac{1}{x}+\frac{1}{1-e^{-x}}
$$

$$
A_{2}^{\prime} \stackrel{\text { def }}{=} A_{2}-A_{2}(0)=-\frac{1}{x}+\frac{1+e^{-x}}{2\left(1-e^{-x}\right)}
$$

Knowing $\tilde{\alpha}_{n, 2}$ we can compute arbitrary $\tilde{\alpha}_{n, m}$ via

$$
\begin{aligned}
\tilde{\alpha}_{n, m} & =\frac{1}{m !} \tilde{\alpha}_{n-1,2}-\tilde{\alpha}_{n-1, m+1} \\
& =\frac{1}{m !} \tilde{\alpha}_{n-1,2}-\frac{1}{(m+1) !} \tilde{\alpha}_{n-2,2}+\cdots+(-1)^{n-1} \frac{1}{(n+m-1) !} \tilde{\alpha}_{0,2}+(-1)^{n} \frac{1}{(m+n) !}
\end{aligned}
$$

We will not write down a closed expression for $\tilde{\alpha}_{n, m}$ as we won't need it.

9.4. Computing a sum. For use below we compute the sum for $n \geq 2$

$$
s_{n}=(-1)^{n-1} \tilde{\alpha}_{n-1,2}+(-1)^{n-2} \tilde{\alpha}_{n-2,3}+\cdots+\tilde{\alpha}_{2, n-1}
$$

where an empty sum is interpreted as 0 . Using (9.3) we get

$$
\begin{gathered}
\tilde{\alpha}_{2, n-1}=\frac{1}{(n-1) !} \tilde{\alpha}_{1,2}-\frac{1}{n !} \tilde{\alpha}_{0,2}+\frac{1}{(n+1) !} \\
\tilde{\alpha}_{3, n-2}=\frac{1}{(n-2) !} \tilde{\alpha}_{2,2}-\frac{1}{(n-1) !} \tilde{\alpha}_{1,2}+\frac{1}{n !} \tilde{\alpha}_{0,2}-\frac{1}{(n+1) !}
\end{gathered}
$$

and

$$
\tilde{\alpha}_{n-1,2}=\frac{1}{2 !} \tilde{\alpha}_{n-2,2}-\frac{1}{3 !} \tilde{\alpha}_{n-3,2}+\cdots+(-1)^{n-2} \frac{1}{n !} \tilde{\alpha}_{0,2}+(-1)^{n-1} \frac{1}{(n+1) !}
$$

so that we get

$$
s_{n}=(-1)^{n-1} \frac{1}{2 !} \tilde{\alpha}_{n-2,2}+(-1)^{n-2} \frac{2}{3 !} \tilde{\alpha}_{n-3,2}+\cdots+(n-2) \frac{1}{(n-1) !} \tilde{\alpha}_{1,2}-(n-2) \frac{1}{n !} \tilde{\alpha}_{0,2}+(n-2) \frac{1}{(n+1) !}
$$

Put

Then we get

$$
T=\frac{x^{2}}{2 !}-\frac{2 x^{3}}{3 !}+\cdots=-x e^{-x}-e^{-x}+1
$$

$$
s_{n}=(-1)^{n-1}\left(T A_{2}\right)\left[x^{n}\right]+\frac{1}{n !} \tilde{\alpha}_{0,2}+\frac{n-2}{(n+1) !}
$$

where $(-)\left[x^{n}\right]$ denotes the coefficient of $x^{n}$. We will regard this formula as a definition if $n=0,1$. We obtain

$$
\sum_{n \geq 0}(-1)^{n} s_{n} x^{n}=-T A_{2}+\sum_{n \geq 0}(-1)^{n} \tilde{\alpha}_{0,2} \frac{x^{n}}{n !}+\sum_{n \geq 0}(-1)^{n} \frac{n-2}{(n+1) !} x^{n}
$$

We have

$$
\sum_{n \geq 0}(-1)^{n} \tilde{\alpha}_{0,2} \frac{x^{n}}{n !}=\frac{1}{2} e^{-x}
$$


and

$$
\begin{aligned}
\sum_{n \geq 0}(-1)^{n} \frac{n-2}{(n+1) !} x^{n} & =\sum_{n \geq 0}(-1)^{n} \frac{n+1}{(n+1) !} x^{n}-3 \sum_{n \geq 0}(-1)^{n} \frac{1}{(n+1) !} x^{n} \\
& =e^{-x}+3 \frac{e^{-x}-1}{x}
\end{aligned}
$$

A computation with a computer algebra package now yields

$$
\sum_{n \geq 0}(-1)^{n} s_{n} x^{n}=\frac{-(x+4) e^{-2 x}+\left(2 x^{2}+3 x+8\right) e^{-x}-(2 x+4)}{2\left(1-e^{-x}\right) x}
$$

Hence

$$
S \stackrel{\text { def }}{=} \sum_{n \geq 0} s_{n} x^{n}=\frac{(x-4) e^{2 x}+\left(2 x^{2}-3 x+8\right) e^{x}+(2 x-4)}{2\left(e^{x}-1\right) x}
$$

We have $S=-3 / 2+O\left(x^{4}\right)$. For use below we record

$$
S^{\prime} \stackrel{\text { def }}{=} \sum_{n \geq 2} s_{n} x^{n}=S+\frac{3}{2}=\frac{(x-4) e^{2 x}+\left(2 x^{2}+8\right) e^{x}-x-4}{2\left(e^{x}-1\right) x}
$$

9.5. Computing $\tilde{\beta}_{n, 1}$. We will only compute $\tilde{\beta}_{n, 1}$ since this the only thing we need. Iterating (9.2) we get for $n \geq 3$

$$
\begin{aligned}
\tilde{\beta}_{n, 1}= & (-1)^{n-1} \frac{1}{2} \tilde{\alpha}_{n-1,2}-\frac{1}{2 \cdot 1 !} \tilde{\beta}_{n-1,1}+\tilde{\beta}_{n-1,2} \\
= & \frac{1}{2}\left((-1)^{n-1} \tilde{\alpha}_{n-1,2}+(-1)^{n-2} \tilde{\alpha}_{n-2,3}+\cdots+\tilde{\alpha}_{2, n-1}\right)- \\
& \quad \frac{1}{2}\left(\frac{1}{1 !} \tilde{\beta}_{n-1,1}+\frac{1}{2 !} \tilde{\beta}_{n-2,1}+\cdots+\frac{1}{(n-2) !} \tilde{\beta}_{2,1}\right)+\tilde{\beta}_{2, n-1} \\
= & \frac{s_{n}}{2}-\frac{1}{2}\left(\frac{1}{1 !} \tilde{\beta}_{n-1,1}+\frac{1}{2 !} \tilde{\beta}_{n-2,1}+\cdots+\frac{1}{(n-2) !} \tilde{\beta}_{2,1}\right)+\tilde{\beta}_{2, n-1}
\end{aligned}
$$

Since $s_{2}=0$ this identity holds for $n=2$ if we interpret an empty sum as zero.

If we put

$$
B_{1}=\sum_{n \geq 2} \tilde{\beta}_{n, 1} x^{n}
$$

then we have

$$
B_{1}=\frac{1}{2} S^{\prime}-\frac{1}{2}\left(\frac{x}{1 !}+\frac{x^{2}}{2 !}+\cdots\right) B_{1}+\sum_{n \geq 2} x^{n}\left(\frac{1}{8(n-1) !}-\frac{1}{2 n !}+\frac{1}{(n+1) !}\right)
$$

We have

$$
\begin{gathered}
\frac{x}{1 !}+\frac{x^{2}}{2 !}+\cdots=e^{x}-1 \\
\sum_{n \geq 2} \frac{x^{n}}{(n-1) !}=x\left(e^{x}-1\right) \\
\sum_{n \geq 2} \frac{x^{n}}{n !}=e^{x}-1-x \\
\sum_{n \geq 2} \frac{x^{n}}{(n+1) !}=\frac{1}{x}\left(e^{x}-1-x-\frac{x^{2}}{2}\right)
\end{gathered}
$$


and hence

$$
\begin{aligned}
\sum_{n \geq 2} x^{n}\left(\frac{1}{8(n-1) !}-\frac{1}{2 n !}+\frac{1}{(n+1) !}\right) & =\frac{1}{8} x\left(e^{x}-1\right)-\frac{1}{2}\left(e^{x}-1-x\right)+\frac{1}{x}\left(e^{x}-1-x-\frac{x^{2}}{2}\right) \\
& =\left(\frac{x}{8}-\frac{1}{2}+\frac{1}{x}\right) e^{x}-\frac{1}{x}-\frac{1}{2}-\frac{x}{8}
\end{aligned}
$$

Thus we get

$$
\left(1+\frac{1}{2}\left(e^{x}-1\right)\right) B_{1}=\frac{1}{2} S^{\prime}+\left(\frac{x}{8}-\frac{1}{2}+\frac{1}{x}\right) e^{x}-\frac{1}{x}-\frac{1}{2}-\frac{x}{8}
$$

Invoking once again a computer algebra package we find

$$
B_{1}=\frac{(x-2) e^{x}+x+2}{4\left(e^{x}-1\right)}
$$

9.6. Computing $w_{n}$. If $n$ is even we have

$$
(-1)^{\frac{n(n-1)}{2}} w_{n}=\tilde{\beta}_{n, 1}-\tilde{\alpha}_{n-1,2}
$$

Since $A_{2}^{\prime}, B_{1}$ are respectively an odd and an even function of $x, B_{1}(0)=0$ and $w_{n}=0$ for $n$ odd we obtain

$$
\sum_{n \geq 2}(-1)^{\frac{n(n-1)}{2}} w_{n} x^{n}=B_{1}-x A_{2}^{\prime}
$$

and using a computer algebra package we find

$$
\sum_{n \geq 2}(-1)^{\frac{n(n-1)}{2}} w_{n} x^{n}=\frac{(x+2) e^{-x}+x-2}{4\left(e^{-x}-1\right)}
$$

The derivative of

is equal to

$$
\frac{1}{2} \log \frac{e^{x / 2}-e^{-x / 2}}{x}
$$

$$
-\frac{(x+2) e^{-x}+x-2}{4\left(e^{-x}-1\right) x}
$$

Dividing (9.4) by $x$ and integrating finishes the proof of (1.1).

\section{REFERENCES}

[1] D. Arnal, D. Manchon, and M. Masmoudi, Choix des signes pour la formalité de M. Kontsevich, Pacific J. Math. 203 (2002), no. 1, 23-66.

[2] D. Calaque and M. Van den Bergh, Hochschild cohomology and Atiyah classes, submitted, arXiv:0708.2725.

[3] A. Cattaneo, B. Keller, C. Torossian, and A. Bruguières, Introduction, Déformation, quantification, théorie de Lie (Paris), Panor. Synthèses, vol. 20, Soc. Math. France, Paris, 2005, Dual French-English text, pp. 1-9, 11-18.

[4] A. S. Cattaneo and G. Felder, Coisotropic submanifolds in Poisson geometry and branes in the Poisson sigma model, Lett. Math. Phys. 69 (2004), 157-175.

[5] Lelative formality theorem and quantisation of coisotropic submanifolds, Adv. Math. 208 (2007), no. 2, 521-548.

[6] GSL - GNU Scientific Library, http://www.gnu.org/software/gsl.

[7] M. Kontsevich, Deformation quantization of Poisson manifolds, Lett. Math. Phys. 66 (2003), no. $3,157-216$.

[8] Maxima, http://maxima.sourceforge.net.

[9] SAGE Mathematical Software, Version 2.6, http://www.sagemath.org.

[10] B. Shoikhet, On the Duflo formula for $L_{\infty}$-algebras and Q-manifolds, math/9812009. 
[11] _ Vanishing of the Kontsevich integrals of the wheels, Lett. Math. Phys. 56 (2001), no. 2, 141-149, EuroConférence Moshé Flato 2000, Part II (Dijon).

[12] E. W. Weisstein, Modified Bernoulli Number, MathWorld, http://mathworld.wolfram.com /ModifiedBernoulliNumber.html.

[13] T. Willwacher A counterexample to the quantizability of modules, Lett. Math. Phys. 81 (2007), no. 3, pp. 265-280.

Departement WNi, Universiteit Hasselt, Universitaire Campus, Building D, 3590 DiepenBEEK, BELGIUM

E-mail address: michel.vandenbergh@uhasselt.be 


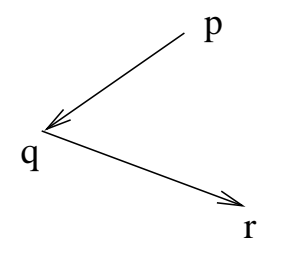




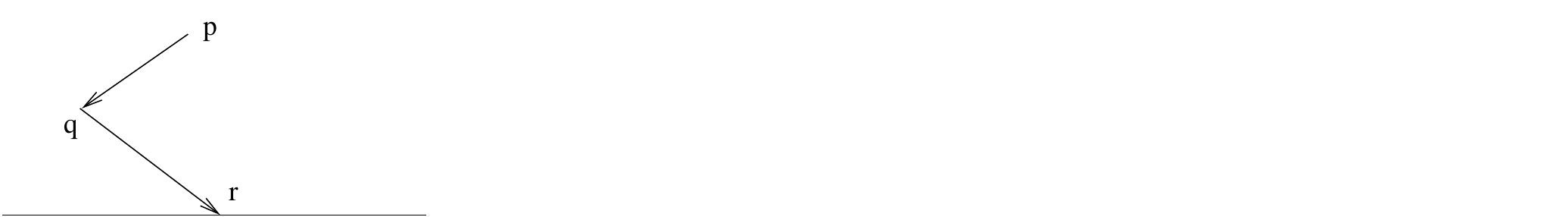




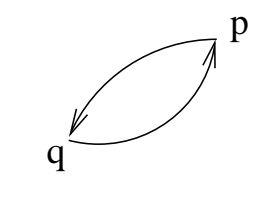


